\title{
1 A Pluripotent Developmental State Confers a Low Fidelity of Chromosome Segregation
}

3 Chenhui Deng ${ }^{1,2},{\text { Amanda } Y a^{1,2} \text {, Duane A. Compton }}^{1,2}$, and Kristina M. Godek ${ }^{1,2, *}$

5 1. Department of Biochemistry and Cell Biology, Geisel School of Medicine at Dartmouth,

6 Hanover, $\mathrm{NH}$, United States of America.

7 2. Norris Cotton Cancer Center, Geisel School of Medicine at Dartmouth, Lebanon, NH, United

8 States of America.

\section{$20{ }^{*}$ Correspondence:}

21 Kristina M. Godek, Ph.D.

22 Geisel School of Medicine at Dartmouth

23 Department of Biochemistry and Cell Biology, HB7650

24 Phone: +1 (603) 646-5192

25 Lebanon, $\mathrm{NH}, 03756$, USA

26 Kristina.M.Godek@dartmouth.edu 


\section{Summary:}

Human pluripotent stem cells (hPSCs) frequently become aneuploid with abnormal

29 chromosome numbers due to mitotic chromosome segregation errors during propagation in

30 culture. Yet, we do not understand why hPSCs exhibit a low mitotic fidelity. Here we investigate

31 the mechanisms responsible for mitotic errors in hPSCs and show that the primary cause is

32 lagging chromosomes with improper merotelic chromosome microtubule attachments in

33 anaphase. Accordingly, we can improve merotelic error correction and reduce lagging

34 chromosome rates in hPSCs using small molecules that prolong mitotic duration or destabilize

35 chromosome microtubule attachments providing chemical strategies to preserve genome

36 stability. Strikingly, we also demonstrate that mitotic error rates correlate with developmental

37 potential decreasing upon differentiation and loss of pluripotency and conversely increasing

38 after reprogramming to a pluripotent state. Thus, chromosome segregation fidelity is inherently

39 low in hPSCs and depends on developmental state in normal human cells.

41 Keywords: Aneuploidy, mitosis, chromosome segregation, human pluripotent stem cells,

42 preimplantation, chromosomal instability, developmental potential 


\section{Introduction:}

Human pluripotent stem cells (hPSCs), including human embryonic stem cells (hESCs) and induced pluripotent stem cells (iPSCs), have the ability to differentiate into cells of all three embryonic germ layers and hence hold great promise for modeling and treating human diseases and conditions. However, during propagation in culture, hPSCs often become aneuploid with abnormal numbers of chromosomes (Baker et al., 2007; Mayshar et al., 2010; Taapken et al., 2011). Aneuploidy in hPSCs is attributed to culture adaptation that selects for abnormal, stable aneuploid karyotypes which outcompete diploid hPSCs limiting potential therapeutic

51 applications (Baker et al., 2007; Keller and Spits, 2021; Mayshar et al., 2010; Price et al., 2021;

52 Taapken et al., 2011).

Although culture adaptation explains how reoccurring constitutive aneuploidies become dominant in cultures of hPSCs, it does not explain how or why mitotic chromosome segregation errors occur in hPSCs generating an aneuploid genome. Perturbed DNA replication dynamics,

DNA damage and defects in chromosome condensation that typically cause structural aneuploidies involving copy number alterations to chromosomal segments are linked to mitotic defects in hPSCs (Burrell et al., 2013; Halliwell et al., 2020; Lamm et al., 2016). However, whole chromosome aneuploidies resulting in the gain or loss of whole chromosomes are also prevalent in hPSCs (Baker et al., 2007; Mayshar et al., 2010; Taapken et al., 2011), but we do not know the mitotic pathways responsible for whole chromosome segregation errors in hPSCs. Similarly, during early human embryogenesis aneuploidy is prevalent in totipotent and pluripotent embryonic cells with aneuploidy rates ranging between $25-90 \%$ for in vitro fertilization (IVF) preimplantation human embryos (Baart et al., 2006; Fragouli et al., 2008, 2013; McCoy et al., 2015; Mertzanidou et al., 2013; Vanneste et al., 2009) making aneuploidy the

66 leading cause of miscarriages and birth defects in humans (Hassold and Hunt, 2001; Menasha

67 et al., 2005; Orr et al., 2015). The high incidence of aneuploidy occurs irrespective of maternal 68 age, infertility or embryo quality (Mertzanidou et al., 2013; Popovic et al., 2019; Vanneste et al., 
69 2009). Due to obvious legal and ethical restrictions, aneuploidy rates in naturally conceived

70 human embryos are unknown but are thought to correspond to aneuploidy rates in IVF

71 preimplantation embryos accounting for a low human fecundity rate with only $\sim 30 \%$ of

72 conceptions resulting in live births (Macklon et al., 2002; McCoy, 2017).

73 Surprisingly, like hPSCs, whole chromosome abnormalities caused by mitotic errors are

74 more frequent than meiotic errors and structural aneuploidies in IVF preimplantation embryos

75 (McCoy et al., 2015; Vanneste et al., 2009). This raises the intriguing possibility that mitotic

76 errors and aneuploidy in hPSCs are not solely an artifact of growth in culture but rather are

77 intrinsic characteristics of pluripotent cells. Yet, like hPSCs, we also do not know the underlying mechanisms causing the persistent and high rate of mitotic chromosome segregation errors in preimplantation embryonic cells. To investigate the mechanisms responsible for mitotic chromosome segregation errors in pluripotent cells, we categorize and quantify mitotic errors in

81 hPSCs using fixed and time-lapse live-cell fluorescence microscopy. Furthermore, we use small

82 molecules and manipulate development potential to test the influence of mitotic duration and

83 chromosome microtubule attachment stability and developmental state, respectively, on

84 chromosome segregation fidelity in hPSCs.

86 Results:

87 Lagging chromosomes, caused by merotelic microtubule attachments, are responsible

88 for elevated mitotic error rates in hPSCs

To determine the mechanisms causing mitotic chromosome segregation errors in

90 hPSCs, we categorized the types of anaphase errors observed and compared anaphase error

91 rates between pluripotent $\mathrm{H} 1$ and $\mathrm{H} 9$ human embryonic stem cells (hESCs) derived from the

92 inner cell mass of human blastocysts (Thomson et al., 1998) and normal, primary somatic BJ

93 fibroblasts (Figures 1A-B). Lagging chromosomes, unaligned chromosomes, and multipolar

94 anaphases cause whole chromosome aneuploidy while acentric DNA fragments and 
95 chromosome bridges lead to structural aneuploidy (Figures 1A-B) (Burrell et al., 2013; Orr et al.,

96 2015; Thompson and Compton, 2008). We also included a combination category for cells that

97 exhibited multiple types of anaphase errors (Figures 1A-B).

$98 \quad$ In somatic BJ fibroblasts, lagging chromosomes were the most frequent anaphase error;

99 however, the rate was less than 5\% (Figure 1B). In agreement, mitotic error and aneuploidy

100 rates are less than 5\% in other normal human somatic cells and tissues (Cimini et al., 1999;

101 Knouse et al., 2014; Thompson and Compton, 2008). Lagging chromosomes were also the

102 most frequent anaphase error in $\mathrm{H} 1$ and $\mathrm{H} 9$ hESCs; however, the rate was significantly higher,

103 more than double (>10\% in $\mathrm{H} 1$ and $\mathrm{H} 9$ hESCs), compared to somatic BJ fibroblasts (Figure 1B).

104 Furthermore, H1 hESCs had a significantly higher frequency of acentric DNA fragments

105 compared to somatic BJ fibroblasts. Though, it was less than half the frequency of lagging

106 chromosomes in H1 hESCs, and there was not a similar trend in H9 hESCs (Figure 1B). In

107 parallel samples, we quantified that more than $95 \%$ of the $\mathrm{H} 1$ or the $\mathrm{H} 9 \mathrm{hESC}$ population

108 expressed the pluripotency transcription factors OCT4 or NANOG demonstrating that

109 spontaneously differentiated cells did not account for the elevated error rates (Figures S1A-B).

110 As lagging chromosomes were the most frequent anaphase error in hESCs (Figure 1B),

111 we sought to determine the causes of these. Using a calcium stable microtubule assay (Warren

112 et al., 2020), we examined chromosome microtubule attachment orientations in hESCs because

113 in other mammalian and human cancer cells, lagging chromosomes are caused by the

114 persistence of improper merotelic chromosome microtubule attachments (also referred to as

115 kinetochore microtubule or k-MT) in anaphase with a chromosome simultaneously attached to

116 microtubules from both spindle poles (Cimini et al., 2001; Thompson and Compton, 2008;

117 Thompson et al., 2010). In metaphase, H1 and H9 hESCs had correct bioriented attachments

118 with sister chromatids attached to microtubules from opposite spindle poles and incorrect

119 merotelic attachments with a single chromatid simultaneously attached to microtubules from

120 both spindle poles (Figures $1 \mathrm{C}$ and S1C). Notably, $73 \%$ of the $\mathrm{H} 1$ and $50 \%$ of the $\mathrm{H} 9$ lagging 
121 chromosomes in anaphase had merotelic attachments (Figures 1C and S1C). We could not

122 reliably conclude the attachment orientations of the remaining lagging chromosomes because in

123 some instances, we observed a lagging chromosome attached to microtubules extending in

124 opposite directions, but we could not track the microtubules back to the spindle poles. Also, the

125 absence of a merotelic attachment may reflect the calcium sensitivity of the attachment rather

126 than an alternative attachment orientation. Because of these limitations, our results likely

127 underestimate the proportion of lagging chromosomes with merotelic attachments in hESCs.

128 HESCs often acquire stable chromosome abnormalities during culturing (Baker et al.,

129 2007; Mayshar et al., 2010; Taapken et al., 2011) that, when coupled with the increased

130 genomic instability caused by an aneuploid genome (Passerini et al., 2016; Sheltzer et al.,

131 2011), suggests the possibility that only aneuploid hESCs exhibit erroneous lagging

132 chromosomes with merotelic attachments. To address this possibility, prior to performing the

133 calcium stable microtubule assay, we karyotyped both $\mathrm{H} 1$ and $\mathrm{H} 9 \mathrm{hESC}$ populations to monitor

134 genomic stability since it is not feasible to simultaneously measure anaphase errors and

135 determine the karyotype of cells. In both the $\mathrm{H} 1$ and the $\mathrm{H} 9$ populations, 20 of 20 cells scored

136 were diploid. From this data, we can infer that less than $14 \%$ of cells in either population are

137 aneuploid with 95\% confidence (Baker et al., 2016) arguing that at least some $\mathrm{H} 1$ and $\mathrm{H} 9$

138 hESCs exhibiting lagging chromosomes with merotelic attachments are diploid.

139 To further validate our findings, we performed time-lapse live-cell fluorescence

140 microscopy using normal, immortalized somatic RPE-1 H2B-GFP epithelial cells, H1 H2B-GFP

141 hESCs (Calder et al., 2013), and AICS-061 human induced pluripotent stem cells (hiPSCs) that

142 were derived from parental WTC-11 hiPSCs reprogrammed from dermal fibroblasts (Hayashi et

143 al., 2016). Somatic RPE-1 H2B-GFP cells and H1 H2B-GFP hESCs exogenously express

144 histone H2B tagged with GFP while AICS-061 hiPSCs express endogenous H2B monoallelically

145 tagged with mEGFP allowing us to quantify anaphase error rates (Figures 1D-E and Videos S1-

146 3). Similar to our previous results, lagging chromosomes were the most frequent anaphase 
147 error, and the rate was significantly elevated in H1 H2B-GFP hESCs (22\% Laminin-521 and

$14821 \%$ Matrigel) and AICS-061 hiPSCs (21\%) compared to somatic RPE-1 H2B-GFP cells (7\%)

149 (Figure 1E and Video S2). Although we cannot definitively distinguish acentric DNA fragments

150 from lagging chromosomes in these experiments, we classified these errors as lagging

151 chromosomes based upon the low incidence of acentrics in all our other analyses (Figures 1B

152 and S3B, S4C and S4E). Thus, a shared phenotype of pluripotent cells is a high mitotic error

153 rate compared to somatic cells with lagging chromosomes being the most frequent error.

154 Furthermore, the lagging chromosome rate was significantly elevated in $\mathrm{H} 1 \mathrm{H} 2 \mathrm{~B}-\mathrm{GFP}$ hESCs

155 compared to somatic RPE-1 H2B-GFP cells irrespective of whether we dissociated and seeded

$156 \mathrm{H} 1 \mathrm{H} 2 \mathrm{~B}-\mathrm{GFP}$ hESCs as single cells without initial cell-cell contacts on a Laminin-521 substrate

157 or as aggregates that maintain cell-cell contacts on a Matrigel substrate (Figure 1E). Our results

158 combined with the high incidence of mitotic errors in IVF preimplantation embryos (McCoy et al.,

159 2015; Vanneste et al., 2009), which maintain their 3D structure, argue that the disruption of

160 tissue structure is unlikely to artificially increase mitotic error rates for hESCs growing in culture

161 in contrast to recent findings in somatic epithelial tissues (Knouse et al., 2018).

162 Chromosome bridges were the second most frequent error, but there was not a

163 significant difference in the chromosome bridge rate between somatic RPE-1 cells and hPSCs

164 (Figure 1E). Also, we rarely observed multipolar anaphases or unaligned chromosomes (1/258

165 each in AICS-061 hiPSCs) (Figure 1E). The low incidence of unaligned chromosomes in hPSCs

166 (Figures 1B and E) indicates the spindle assembly checkpoint (SAC) is functionally preventing

167 anaphase onset until microtubules attach to chromosomes, which facilitates chromosome

168 alignment (Musacchio and Salmon, 2007). In further support, we observed an H1 H2B-GFP

169 hESC that delayed anaphase onset for more than 2 hrs due to a chromosome that failed to align

170 (Figure S1D and Video S4), and hESCs arrest in mitosis in the presence of the microtubule

171 depolymerizing drug nocodazole (Becker et al., 2006; Zhang et al., 2019). Thus, SAC signaling

172 is functional and responsive to unattached chromosomes in hPSCs. In contrast, mouse morulae 
173 stage embryonic cells exhibit a high frequency of unaligned chromosomes indicative of

174 insufficient SAC signaling (Vázquez-Diez et al., 2019). Collectively, our results agree with

175 previous studies that quantified total mitotic error rates between 15-20\% in hPSCs (Halliwell et

176 al., 2020; Lamm et al., 2016; Milagre et al., 2020; Peterson et al., 2011; Taapken et al., 2011;

177 Zhang et al., 2019), but importantly we extend these observations and demonstrate that lagging

178 chromosomes in anaphase, caused by improper merotelic attachments, are the most frequent

179 mitotic error in hPSCs and that lagging chromosome rates are significantly elevated in hPSCs

180 compared to somatic cells.

\section{Prolonging mitotic duration decreases mitotic error rates in hPSCs}

Chromosome missegregation rates are proportional to lagging chromosome rates because lagging chromosomes with merotelic attachments have an increased likelihood of segregating to the incorrect daughter cell producing two aneuploid progeny (Thompson and because culture adaptation that selects for hPSCs with abnormal, stable aneuploid karyotypes must initiate with mitotic errors to generate the aneuploid substrates for selection (Baker et al.,

1912007 ; Taapken et al., 2011). Improper merotelic attachments are not detected by the SAC (Cimini et al., 2001) but

193 instead a network of kinases (Cimini et al., 2006; Godek et al., 2014; Salimian et al., 2011) and

194 microtubule depolymerases (Bakhoum et al., 2008; Godek et al., 2014) converts improper

195 merotelic attachments to correct bioriented attachments by facilitating iterative cycles of

196 microtubule detachment and reattachment prior to anaphase onset (Godek et al., 2014). Thus,

197 one parameter that influences merotelic error correction efficiency is mitotic duration with a

198 longer mitotic duration allowing for more cycles of microtubule detachment and reattachment 
199 decreasing the frequency of errors (Figure 2A, note: error correction rate does not change)

200 (Cimini et al., 2003; Sansregret et al., 2017) and conversely a shorter mitotic duration increasing

201 errors. Accordingly, if mitotic duration is insufficient for robust merotelic error correction in

202 hPSCs this will cause an elevated frequency of lagging chromosomes. A prediction of this

203 hypothesis is that mitotic duration is shorter in hPSCs than somatic cells.

204 To test this hypothesis, we measured mitotic duration from nuclear envelope breakdown

205 (NEB) to anaphase onset (AO) in the H1 H2B-GFP hESCs, AICS-061 hiPSCs, and somatic

206 RPE-1 H2B-GFP cells that we quantified anaphase error rates in (Figure 1E). In both H1 H2B-

207 GFP hESCs and AICS-061 hiPSCs mitotic duration from NEB to AO, including prometaphase

208 (NEB to metaphase) and metaphase (metaphase to AO), was significantly increased compared

209 to somatic RPE-1 cells demonstrating that the elevated lagging chromosome rates in hPSCs

210 are not caused by an abbreviated mitotic duration compared to somatic cells (Figure S1E). In

211 further support, there was no significant difference in mitotic duration, including prometaphase

212 or metaphase, between hPSCs that went through a normal mitosis or an aberrant mitosis

213 (Figure S1F) underscoring that an abbreviated mitosis does not account for errors. These

214 results suggest that other mechanisms are responsible for the elevated frequency of lagging

215 chromosomes in hPSCs compared to somatic cells (see next section).

$216 \quad$ Nevertheless, we tested if prolonging mitosis would effectively reduce lagging

217 chromosome rates in hPSCs by allowing for more cycles of microtubule release and

218 reattachment prior to anaphase onset (Figure 2A). To test this strategy, we used the small

219 molecule proTAME to delay mitotic progression. ProTAME inhibits the anaphase promoting

220 complex/cyclosome (APC/C) E3 ubiquitin ligase whose activity is required for mitotic exit and

221 whose partial inhibition increases mitotic duration in human somatic and cancer cells (Zeng et

222 al., 2010). As a positive control, we reproduced previous results demonstrating that prolonging

223 mitosis with proTAME reduces the frequency of mitotic errors, including lagging chromosomes,

224 in somatic RPE-1 cells when error rates are artificially elevated (Figures S2A-C) (Sansregret et 
225 al., 2017). For our experiments in hPSCs, we added proTAME to H1 H2B-GFP hESCs or AICS226061 hiPSCs immediately prior to starting time-lapse live-cell imaging and imaged cells for 7 hrs 227 in the presence of proTAME. Also, in parallel samples, we quantified that more than $95 \%$ of the $228 \mathrm{H} 1 \mathrm{H} 2 \mathrm{~B}-\mathrm{GFP}$ or the AICS-061 hPSCs expressed OCT4 or NANOG prior to proTAME treatment, 229 indicating that spontaneously differentiated cells in the populations were unlikely to influence the 230 outcomes (Figures S2G and J).

231 In both H1 H2B-GFP hESCs and AICS-061 hiPSCs, mitotic duration significantly

232 increased proportionally with proTAME concentration (Figures $2 \mathrm{~B}-\mathrm{C}$ and $\mathrm{S} 2 \mathrm{H}$ ). Importantly, as

233 mitotic duration increased, the incidence of lagging chromosomes significantly decreased for $\mathrm{H} 1$

234 H2B-GFP hESCs (Figures 2C-D). The chromosome bridge and total anaphase error rates also 235 significantly decreased with proTAME treatment (Figures 2C-D and S2E). Chromosome bridges 236 are a consequence of under-replicated DNA regions or unresolved aberrant DNA structures that

237 persist into mitosis and prolonging mitosis may also facilitate the correction of these errors

238 (Fragkos and Naim, 2017). Surprisingly, the frequency of lagging chromosomes did not

239 significantly decrease in AICS-061 hiPSCs (Figure S2I) despite an increased mitotic duration 240 comparable to H1 H2B-GFP hESCs (Figures 2C and S2H). Consequently, we checked the 241 genomic stability of AICS-061 hiPSCs during these experiments reasoning that aneuploid cells

242 could be insensitive to this approach. There was a clonal abnormal karyotype, including a

243 terminal deletion of the long arm of chromosome 18, but it was present at a low frequency in the 244 population $(10 \%, 2 / 20)$ and thus is unlikely to be the reason for the different response. We

245 speculate that in AICS-061 hiPSCs other parameters have a greater influence on merotelic error 246 correction. Also, we note that the lagging chromosome rate $(\sim 20 \%)$ is approximately double the

247 frequency of aneuploid cells in the population indicating that aneuploid cells do not solely

248 account for the error rate.

249 The reduction in the lagging chromosome rate in $\mathrm{H} 1 \mathrm{H} 2 \mathrm{~B}-\mathrm{GFP}$ hESCs could be a 250 consequence of prolonging prometaphase, metaphase or both. Interestingly, metaphase was 
251 selectively lengthened proportional to proTAME concentration (Figure S2D) in H1 H2B-GFP

252 hESCs, similar to somatic RPE-1 H2B-GFP cells (Figure S2A), demonstrating that, at least for

253 some cells, metaphase duration can be a rate-limiting step in merotelic error correction. In

254 further support, there was a significant decrease in metaphase duration for H1 H2B-GFP hESCs

255 that went through mitosis with a lagging chromosome vs. a normal mitosis in the $20 \mu \mathrm{M}$

256 proTAME treatment group (Figure S2F). However, this trend did not occur in the $6 \mu \mathrm{M}$ proTAME

257 treatment group (Figure S2F) revealing that error correction is not exclusively limited by

258 metaphase duration. Combined, our results demonstrate that the elevated frequency of lagging

259 chromosomes in hPSCs compared to somatic cells is not caused by an abbreviated mitosis;

260 however, delaying mitotic progression, and metaphase specifically, is an effective strategy to

261 improve merotelic error correction and reduce the lagging chromosome rate in hPSCs, albeit

262 with the application limited to select hPSC lines.

Decreasing microtubule attachment stability reduces mitotic errors in hPSCs

The iterative cycles of microtubule detachment and reattachment required for merotelic

266 error correction also dictate that the error correction rate depends on chromosome microtubule

267 attachment turnover with hyperstable attachments (i.e. low turnover) inhibiting the release of

268 incorrect merotelic attachments (Bakhoum et al., 2009; Godek et al., 2014). Hence, hyperstable

269 chromosome microtubule attachments in hPSCs relative to somatic cells is an alternative

270 hypothesis explaining the elevated incidence of lagging chromosomes in hPSCs. This predicts

271 that decreasing microtubule attachment stability (i.e. increasing turnover) will reduce lagging

272 chromosome rates in hPSCs (Figure 3A, note: mitotic duration does not change).

273 To test this prediction, we used the small molecule UMK57, an agonist of the

274 microtubule depolymerase mitotic centromere-associated kinesin (MCAK or KIF2C), to

275 decrease microtubule attachment stability in hPSCs. In human cancer cells with hyperstable 
276 attachments, short-term UMK57 treatment potentiates MCAK activity destabilizing microtubule

277 attachments in metaphase and thus reduces the lagging chromosome rate (Orr et al., 2016).

278 We treated $\mathrm{H} 1$ and $\mathrm{H} 9$ hESCs and positive control U2OS cancer cells for 45 mins with UMK57

279 prior to measuring anaphase error rates. To control for off-target effects, we also measured

280 errors in cells treated for 45 mins with UMK95, an inactive analog of UMK57 (Orr et al., 2016).

281 As expected, short-term UMK57 treatment in U2OS cancer cells significantly reduced

282 the lagging chromosome rate while UMK95 treatment did not (Figure 3B) (Orr et al., 2016).

283 Likewise, lagging chromosome rates were significantly reduced by approximately $50 \%$ in $\mathrm{H} 1$

284 and H9 hESCs treated with UMK57, but at higher concentrations, while UMK95 treatment did

285 not (Figure 3B). Also, lagging chromosome rates were selectively reduced while other anaphase

286 error rates were not (Figure 3B and S3B), highlighting that the mechanisms responsible for

287 different types of mitotic errors are distinct. Furthermore, unlike in cancer cells, high doses of

288 UMK57 did not affect $\mathrm{H} 1$ or H9 hESC mitotic progression (Figure S3A) (Orr et al., 2016). As

289 previous, we determined that spontaneously differentiated cells in the $\mathrm{H} 1$ and the $\mathrm{H} 9$

290 populations did not account for the error rates (Figure S3E). Moreover, we performed these

291 experiments using the same batch of $\mathrm{H} 1$ and $\mathrm{H} 9$ hESCs that we karyotyped for the calcium

292 stable microtubule assay and showed were diploid within the sensitivity range for the number of

293 cells scored. Combined, these results demonstrate that destabilizing chromosome microtubule

294 attachments in hPSCs increases the rate of merotelic error correction, reducing the frequency of

295 lagging chromosomes (Figure 3A).

296 Although we modeled the effects of mitotic duration and chromosome microtubule

297 attachment stability on merotelic error correction as two separate and independent pathways

298 (Figures 2A and 3A), these may influence error correction in a dependent manner. To test this

299 possibility, we simultaneously measured mitotic duration and errors in H1 H2B-GFP hESCs and

300 AICS-061 hiPSCs by time-lapse live-cell fluorescence microscopy in the presence of UMK57 for

$30112 \mathrm{hrs}$ (Figures 3C-D and S3C-D). In contrast to prolonging mitosis with proTAME (Figures S2I 
302 and J), destabilizing microtubule attachments with UMK57 significantly reduced lagging

303 chromosome rates in both H1 H2B-GFP hESCs (Figure 3C) and AICS-061 hiPSCs (Figure 3D)

304 while UMK95 did not. The chromosome bridge rate also significantly decreased in H1 H2B-GFP

305 hESCs (Figure 3C), but this was not consistent in the AICS-061 hiPSCs (Figure 3D).

306 Interestingly, there was a significant increase in mitotic duration with UMK57 treatment,

307 and specifically metaphase, for both H1 H2B-GFP hESCs and AICS-061 hiPSCs while UMK95

308 treatment did not significantly affect it (Figures S3C-D). However, for H1 H2B-GFP hESCs, the

309 increase in metaphase duration was comparable to $3 \mu \mathrm{M}$ proTAME treatment (metaphase mean

$310=15.8$ mins DMSO vs. 27.3 mins $3 \mu \mathrm{M}$ proTAME and metaphase mean $=12.2$ mins DMSO vs.

31120.4 mins UMK57), which did not significantly reduce the lagging chromosome rate (Figure 2D).

312 For AICS-061 hiPSCs, no amount of delay in mitotic progression reduced the lagging

313 chromosome rate (Figure S2I) suggesting that potentiating MCAK depolymerase activity

314 predominantly enhances error correction by destabilizing microtubule attachments. Thus, mitotic

315 duration and chromosome microtubule attachment stability are largely two independent

316 parameters that influence merotelic error correction efficiency.

317 During these experiments, we also monitored the genomic stability of H1 H2B-GFP

318 hESCs and AICS-061 hiPSCs. Similar to our previous analysis, there were clonal aneuploid

319 cells with a terminal deletion of the long arm of chromosome 18 present in the AICS-061

320 population at a low frequency $(<10 \%, 3 / 32)$. For the $\mathrm{H} 1 \mathrm{H} 2 \mathrm{~B}-\mathrm{GFP}$ hESCs, initial karyotyping

321 done after performing two complete experimental sets found 20 of 20 cells were diploid.

322 Subsequent karyotyping, after the third experimental set, identified a fraction of abnormal cells

323 with an interstitial duplication of the long arm of chromosome 20 in the population $(25 \%, 5 / 20)$.

324 Overall, the reduction in lagging chromosome rates upon UMK57 treatment is reproducible

325 using multiple different hPSC lines arguing that the low incidence of aneuploid cells is unlikely to

326 influence the outcomes. Collectively, these results support our hypothesis that hyperstable 
327 chromosome microtubule attachments contribute to the elevated frequency of erroneous lagging

328 chromosomes in both hESCs and hiPSCs compared to somatic cells and that decreasing

329 microtubule attachment stability is an effective strategy to reduce lagging chromosome rates in

330 hPSCs.

Developmental potential influences mitotic error rates

335 chromosome segregation is conserved in normal, non-transformed cells. Consequently, our repeated observations that mitotic error rates, and particularly lagging chromosome rates, are elevated in hPSCs compared to somatic cells (Figures 1B and D) coupled with the high mitotic error rates in preimplantation human embryos (McCoy et al., 2015; Vanneste et al., 2009) challenge this assumption. The opposing phenotypes of somatic vs. embryonic cells and hPSCs with respect to the frequency of mitotic errors led us to question whether a high error rate is an

341 intrinsic and a cell autonomous trait linked to developmental state. This idea predicts that mitotic

342 error rates and developmental potential correlate such that as developmental potential

343 decreases mitotic error rates decrease and that as developmental potential increases so do

344 mitotic error rates (Figure 4A). We tested this prediction using isogenic cells with different

345 developmental states to eliminate genetic diversity as a confounding variable.

346 We compared mitotic error rates between isogenic normal, primary somatic WTC-11

347 fibroblasts to parental WTC-11 and the derivative AICS-061 hiPSCs. In agreement with our

348 prediction, anaphase errors, with lagging chromosomes being the most frequent error, were

349 significantly elevated in WTC-11 (lagging = 24\%) and AICS-061 hiPSCs (lagging = 23\%)

350 compared to isogenic somatic WTC-11 fibroblasts (lagging $=3 \%$ ) (Figure 4B and S4C). In

351 addition, we karyotyped somatic WTC-11 fibroblasts and WTC-11 hiPSCs to confirm that

352 abnormal aneuploid cells present in either population did not exclusively account for the error 
rates. Somatic WTC-11 fibroblasts were diploid (20/20) while 10\% (2/20) of the WTC-11 hiPSCs

354 had a clonal balanced translocation between the short arm of chromosome 1 and long arm of

355 chromosome 16; however, even with the hypothetical assumption that all aneuploid cells go

356 through an aberrant mitosis with a lagging chromosome and discarding $10 \%$ of the lagging

357 chromosome data, lagging chromosome rates remained significantly elevated in WTC-11

358 hiPSCs compared to somatic WTC-11 fibroblasts (Figure S4D). Furthermore, to confirm the

359 developmental states of isogenic WTC-11 and AICS-061 hiPSCs and somatic WTC-11

360 fibroblasts, we quantified the percent of cells expressing the pluripotency transcription factors

361 OCT4 and NANOG. As expected, somatic WTC-11 fibroblasts did not express OCT4 and

362 NANOG while nearly $100 \%$ of the WTC-11 and AICS-061 hiPSCs did (Figure S4B). Thus, with

363 increased developmental potential mitotic error rates also increase.

364 If mitotic error rates correlate with developmental potential as we predict (Figure 4A),

365 then differentiation and loss of pluripotency should decrease error rates. To test this, we

366 induced undirected differentiation in $\mathrm{H} 1$ or H9 hESCs with all-trans retinoic acid (RA) (Jain et al.,

367 2012). During a 4-day time course, DMSO treated control H1 and H9 hESCs maintained their

368 pluripotent stem cell morphology of tightly packed colonies with smooth borders and a high

369 nuclear to cytoplasmic ratio while RA treated hESCs acquired a flattened morphology and lower

370 nuclear to cytoplasmic ratio (Figure S4F) indicative of differentiation. Also, expression of the

371 pluripotency transcription factors OCT4, NANOG and SOX2 significantly decreased in the RA

372 treated cells at the endpoint comparable to levels in somatic WTC-11 fibroblasts (Figures S5A-

373 C) indicating loss of pluripotency. Importantly, after 4 days of RA undirected differentiation,

374 anaphase error rates, including lagging chromosomes, were significantly decreased by

375 approximately $50 \%$ compared to DMSO control H1 or H9 hESCs (Figures 4C and S4E)

376 demonstrating that decreasing developmental potential reduces mitotic error rates. We also

377 observed a slight, but significant increase, in multipolar anaphases; however, the frequency was

378 less than 3\% (Figure S4E). 
In chimeric mouse embryos and human gastruloids composed of mixed populations of

380 diploid and aneuploid cells, aneuploid cells are depleted as development progresses and

381 differentiation occurs (Bolton et al., 2016; Yang et al., 2021). Analogous to this is the possibility

382 that aneuploid cells present in the starting $\mathrm{H} 1$ and $\mathrm{H} 9$ populations used for the RA experiments

383 are responsible for the mitotic errors but become depleted during differentiation thus decreasing

384 the error rate. This scenario requires that $\mathrm{H} 1$ and $\mathrm{H} 9 \mathrm{hESC}$ populations are composed of

385 aneuploid cells or are mosaic populations of diploid and aneuploid cells. Therefore, we

386 karyotyped the $\mathrm{H} 1$ and the $\mathrm{H} 9 \mathrm{hESC}$ populations after completion of all experimental replicates

387 reasoning that clonal and/or non-clonal aneuploidies were most likely to be detected after

388 prolonged culturing. Critically, both the $\mathrm{H} 1$ and $\mathrm{H} 9$ populations were diploid (20/20) arguing that

389 depletion of aneuploid cells during differentiation is unlikely to explain the decrease in anaphase

390 errors. Collectively, our results show that mitotic error rates correlate with developmental

391 potential and suggest that a high mitotic error rate is an inherent and cell autonomous trait of

392 hPSCs.

\section{Discussion:}

Here we show that lagging chromosomes in anaphase, caused by persistent improper

396 merotelic chromosome microtubule attachments, are the most frequent mitotic error in hPSCs.

397 Surprisingly, our results reveal that hPSCs are more similar to transformed human cancer cells

398 than non-transformed normal somatic cells with respect to mitotic error rates, particularly

399 lagging chromosome rates (Cimini et al., 2001; Godek et al., 2016; Thompson and Compton,

400 2008). Furthermore, we show that mitotic error rates correlate with developmental potential

401 decreasing upon loss and increasing upon gain, demonstrating that a high mitotic error rate is

402 intrinsic to hPSCs. In agreement, multipotent neural stem cells exhibit an intermediate error rate

$403(\sim 10 \%)$ between hPSCs and somatic cells suggesting a linear correlation with developmental

404 potential (Godek et al., 2016). Collectively, these results demonstrate that a high fidelity of 
405 chromosome segregation is not universally conserved in normal, diploid human cells and that it

406 depends on developmental state. This raises the possibility that in cancer cells the

407 (re)acquisition of a developmental program with greater potency rather than of mutations in

408 mitotic genes causes an elevated mitotic error rate in agreement with the low frequency of

409 genetic alterations found in mitotic genes (Greenman et al., 2007; Nath et al., 2015).

Assuming that the chromosome missegregation and lagging chromosome rates are

411 proportional in hPSCs, analogous to cancer cells (Thompson and Compton, 2008), then lagging

412 chromosomes are a leading cause of aneuploidy in hPSCs. In hPSCs, the $\sim 20 \%$ lagging

413 chromosome rate is comparable to that of HT29 colon cancer cells which corresponds to a

$414 \sim 0.3 \%$ missegregation rate per chromosome (Thompson and Compton, 2008). Using this

415 benchmark, we estimate that hPSCs missegregate a chromosome every tenth division. We

416 assume that missegregation would be random as there is no known bias to preferentially

417 missegregate a chromosome in unperturbed conditions. Ideally, we would directly measure

418 chromosome missegregation rates, but the growth of hPSCs as tightly packed colonies

419 combined with their poor survival as single cells poses challenges to using conventional

420 techniques (Godek and Compton, 2018; Thompson and Compton, 2008).

$421 \quad$ Although we estimate a high chromosome missegregation rate, we detect a low

422 frequency of aneuploid hPSCs in culture, indicating that most aneuploid hPSCs are at a

423 selective disadvantage, thus maintaining a predominately diploid population. In this regard,

424 hPSCs resemble somatic cells which arrest in the subsequent cell cycle following chromosome

425 missegregation preserving a homogeneous diploid karyotype (Thompson and Compton, 2010).

426 In contrast, cancer cells tolerate and propagate with aneuploid genomes (Godek et al., 2016;

427 Thompson and Compton, 2010). Alternatively, our estimate may be an overestimate, and the

428 generation of aneuploid progeny is a rarer event in hPSCs. Regardless of the exact

429 missegregation rate, these results delineate a pathway driving the process of culture adaptation

430 in hPSCs that selects for reoccurring stable chromosome abnormalities which do outcompete 
431 diploid hPSCs (Baker et al., 2007; Mayshar et al., 2010; Taapken et al., 2011). We propose the

432 process depends on a lagging chromosome that leads to chromosome missegregation

433 producing aneuploid progeny which are then substrates for culture selection pressures to act on

434 (Figure 5A). In this scenario, lagging chromosomes, which are an inherent and cell autonomous

435 trait, are the key agents of change fueling culture adaptation, but this must also be coupled to

436 the transient survival of aneuploid hPSCs providing an opportunity for selection to occur. How

437 hPSCs gain initial or transient tolerance to an aneuploid genome is unknown, but hPSCs often

438 acquire p53 mutations (Merkle et al., 2017) and this may lead to aneuploidy tolerance as shown

439 in cancer cells (Thompson and Compton, 2010). Subsequently, selection for aneuploid hPSCs

440 with constitutive stable chromosome abnormalities that support long-term survival and

441 propagation with a growth advantage over diploid hPSCs occurs (Price et al., 2021). This multi-

442 step process also explains why culture adaptation often arises during extended culturing (Baker

443 et al., 2007).

444 Given the causal relationship between lagging chromosomes and chromosome

445 missegregation combined with the potential consequences of generating aneuploid progeny,

446 understanding why merotelic attachments persist in hPSCs and devising strategies to reduce

447 merotelic errors is paramount for the successful use of hPSCs in regenerative medicine

448 therapies. Here we find that prolonging mitosis or destabilizing chromosome microtubule

449 attachments using the small molecules proTAME or UMK57, respectively, improves merotelic

450 error correction reducing lagging chromosomes in hPSCs. We note that prolonging mitosis

451 using proTAME also decreases the incidence of unaligned chromosomes during mouse

452 preimplantation development presumably by increasing attachment formation rather than

453 merotelic error correction (Vázquez-Diez et al., 2019), suggesting that this strategy is broadly

454 applicable. By extension we predict that these strategies should also suppress aneuploidy rates

455 in hPSCs, although this remains to be tested. Of interest will be to test long-term UMK57

456 treatment in hPSCs as cancer cells, but not normal dermal fibroblasts (Barroso-Vilares et al., 
457 2020), become resistant to treatment (Orr et al., 2016). Also, it remains unknown if hPSCs

458 maintain pluripotency during long-term treatment with these small molecules.

Furthermore, our UMK57 results suggest that, similar to cancer cells (Bakhoum et al.,

460 2009), hyperstable microtubule attachments underlie the elevated frequency of lagging

461 chromosomes in hPSCs. Measurement of microtubule attachment turnover rates in hPSCs will

462 be necessary to test this. Although many molecular players regulating microtubule dynamics are

463 known (Godek et al., 2014), how these networks differ between somatic cells and cancer cells

464 or hPSCs is unknown. In contrast to aneuploid cancer cells where genetic and transcriptional

465 heterogeneity is a confounding variable (Stingele et al., 2012; Zhao et al., 2019), hPSCs may

466 offer a more tractable system to determine the molecular pathways causing hyperstable

467 microtubule attachments as lagging chromosomes are not exclusive to aneuploid hPSCs.

471 unaligned chromosomes are the most frequent mitotic error (Vázquez-Diez et al., 2019)

472 suggesting different mechanisms are responsible for chromosome missegregation in mouse vs.

473 human embryogenesis. This difference may contribute to the discrepancy in aneuploidy rates

474 with 5\% of mouse embryos (Hassold and Hunt, 2001; Lightfoot et al., 2006; Wei et al., 2011)

475 and 25-90\% of human embryos exhibiting aneuploidy (Baart et al., 2006; Fragouli et al., 2008,

476 2013; McCoy et al., 2015; Mertzanidou et al., 2013; Vanneste et al., 2009). In addition, IVF

477 preimplantation embryos exhibit the related phenomena of chromosomal instability (CIN) that

478 requires (1) persistent chromosome missegregation coupled with (2) the survival and

479 propagation of aneuploid progeny (Orr et al., 2015; Thompson and Compton, 2008, 2010)

480 producing heterogeneous aneuploid cells in a single embryo (Mertzanidou et al., 2013;

481 Vanneste et al., 2009). Although a recent study of preimplantation bovine embryos, models for

482 human embryogenesis, found that a failure of parental pronuclei to properly cluster and 
483 condense their chromosomes led to an increase in errors (Cavazza et al., 2021), parental

484 genome clustering is unique to the first mitotic division and thus cannot account for the repeated

485 mitotic errors that must occur to generate embryos with a CIN phenotype. Rather, erroneous

486 lagging chromosomes are not restricted to specialized mitotic divisions and thus provide a

487 mechanism for the CIN phenotype of human preimplantation embryos (Mertzanidou et al., 2013;

488 Vanneste et al., 2009). Furthermore, although we estimate that every tenth division in hPSCs

489 generates aneuploid progeny, this may underestimate the chromosome missegegration rate in

490 human embryos given the prevalence of CIN in IVF cleavage stage embryos indicating repeated

491 mitotic errors occurring within a few divisions (Mertzanidou et al., 2013; Vanneste et al., 2009).

492 Future investigations, using other model systems for human preimplantation development, will

493 be necessary to determine if the same mechanisms are responsible for mitotic errors as in

494 hPSCs.

The CIN phenotype of preimplantation embryos also requires at least an initial tolerance

to an aneuploid genome. How this occurs and whether a similar mechanism supports a limited

497 tolerance to an aneuploid genome in hPSCs (providing an opportunity for culture selection to

498 occur) is unknown. Accordingly, this raises the question of how euploid embryos are established

499 to support normal development. Like most aneuploid hPSCs, aneuploid preimplantation

500 embryonic cells may be at a selective disadvantage when in competition with diploid embryonic

501 cells. In support, some mosaic blastocysts composed of diploid and aneuploid cells were

502 euploid 12 days post-fertilization (Popovic et al., 2019) and transferred mosaic IVF embryos can

503 result in normal development and live births (Yang et al., 2021). Importantly, our results suggest

504 that the establishment of euploid embryos is also supported by declining mitotic error rates as

505 developmental potential decreases and differentiation occurs (Figure 5B). Thus, during human

506 development genome stability is achieved because the time window comprising embryonic cells

507 with high developmental potency and high mitotic error rates is limited. In contrast, the time

508 window is unlimited for hPSCs growing in culture. In conclusion, we propose that in normal 
509 human cells developmental state differentially influences the fidelity of chromosome segregation

510 and the response to aneuploidy.

\section{Experimental Procedures:}

\section{Cell Lines}

Primary BJ fibroblasts (CRL-2522) and U2OS (HTB-96) cell lines used in this study are

515 available from the American Type Culture Collection (ATCC). We generated RPE-1 cells stably

516 expressing H2B-GFP using parental RPE-1 (CRL-4000) cells available from ATCC. H1/WA01

517 and H9/WA09 hESCs are available from WiCell Research Institute. WTC-11 (GM25256) and

518 AICS-061 hiPSCs are available from the Coriell Institute for Medical Research and Allen

519 Institute for Cell Science, respectively. H1 H2B-GFP hESCs used in this study were obtained

520 from Dr. Jonathan S. Draper, McMaster University. H1 H2B-GFP hESCs also express a G1

521 reporter, but we did not monitor G1 phase in our experiments. WTC fibroblasts were obtained

522 the Gladstone Stem Cell Core.

\section{Cell Culture}

U2OS (XX) cells were grown in Dulbecco's Modified Eagle's Medium (DMEM)

526 supplemented with $10 \%$ fetal calf serum (FCS), $50 \mathrm{U} / \mathrm{mL}$ penicillin and $50 \mu \mathrm{g} / \mathrm{mL}$ streptomycin

527 and $250 \mu \mathrm{g} / \mathrm{L}$ Amphotericin B. RPE-1 H2B-GFP (XX) cells were grown in DMEM supplemented

528 with $10 \%$ fetal calf serum (FCS), $50 \mathrm{U} / \mathrm{mL}$ penicillin and $50 \mu \mathrm{g} / \mathrm{mL}$ streptomycin, $250 \mu \mathrm{g} / \mathrm{L}$

529 Amphotericin B, $20 \mathrm{mM}$ HEPES and $5 \mu \mathrm{g} / \mathrm{ml}$ blasticidin. BJ fibroblast (XY) cells were grown in

530 Eagle's Minimum Essential Medium (EMEM) supplemented with 10\% fetal bovine serum (FBS)

531 and $100 \mathrm{U} / \mathrm{mL}$ penicillin and $100 \mu \mathrm{g} / \mathrm{mL}$ streptomycin. WTC fibroblast (XY) cells were grown in

532 DMEM supplemented with 10\% FBS, 2 mM GlutaMAX-1 (ThermoFisher \#35050061), $0.1 \mathrm{mM}$

533 MEM nonessential amino acids and $100 \mathrm{U} / \mathrm{mL}$ penicillin and $100 \mu \mathrm{g} / \mathrm{mL}$ streptomycin. H1/WA01 
534 hESCs (XY), H9/WA09 hESCs (XX), WTC-11 hiPSCs (XY) and AICS-061 hiPSCs (XY) were 535 grown in mTeSR1 medium (StemCell Technologies \#85870). H1 H2B-GFP hESCs (XY) were 536 grown in mTeSR1 supplemented with $1 \mu \mathrm{g} / \mathrm{mL}$ puromycin. All pluripotent stem cell lines were 537 routinely grown on hESC qualified Matrigel (Corning \#354277). For routine passaging, $\mathrm{H} 1, \mathrm{H} 9$ 538 and H1 H2B-GFP hESCs and WTC-11 hiPSCs were dissociated using versene according to 539 WiCell or Coriell Institute protocols, respectively. AICS-061 hiPSCs were passaged using 540 StemPro Accutase (ThermoFisher \#A1110501) in the presence of ROCK inhibitor Y-27632

541 (Tocris \#1254) for an initial $\sim 20 \mathrm{hrs}$ according to Allen Institute protocols. WTC-11 fibroblasts

542 were routinely passaged using TrypLE Select (ThermoFisher \#12563011), and BJ fibroblasts, 543 U2OS and RPE-1 H2B-GFP cells were passaged using $0.05 \%$ trypsin. All cell lines were 544 validated as mycoplasma free (Sigma-Aldrich Lookout $\circledast$ Mycoplasma PCR Detection Kit \# 545 MP0035) and grown at $37^{\circ} \mathrm{C}$ in a humidified atmosphere with $5 \% \mathrm{CO}_{2}$. The karyotypes of 546 human pluripotent stem cell lines and isogenic WTC fibroblasts used in this manuscript were 547 verified with by G-banded karyotyping provided by WiCell Research Institute.

RPE-1 H2B-GFP cells were transfected with the pBOS H2B-GFP vector (BD

549 Biosciences) using Fugene 6 (Promega \#E2691) following manufacturer's instructions. RPE-1

550 cells stably expressing H2B-GFP were selected using $5 \mu \mathrm{g} / \mathrm{ml}$ blasticidin and subsequently

551 single cell clones were isolated using limiting dilution.

Immunofluorescence

$\mathrm{H} 1$ and $\mathrm{H} 9 \mathrm{hESC}$ and WTC-11 hiPSCs were plated as aggregates on Matrigel-coated

$55518 \mathrm{~mm}$ glass coverslips in 12-well cell culture plates unless otherwise noted in the figure

556 legends. Alternatively, $\mathrm{H} 1$ and H9 hESCs were dissociated to single cells using TrypLE Select 557 and plated on Laminin-521 (Biological Industries \#05-753-1F) coverslips coated at $0.5 \mu \mathrm{g} / \mathrm{cm}^{2}$. 558 AICS-061 hiPSCs were dissociated to single cells using StemPro Accutase and plated on 
Matrigel-coated $18 \mathrm{~mm}$ glass coverslips in 12-well cell culture plates with ROCK inhibitor for an

560 initial $\sim 20 \mathrm{hrs}$ and then subsequently the ROCK inhibitor was washed out. For AICS-061

561 hPSCs, all experiments were performed at least $24 \mathrm{hrs}$ after the removal of ROCK inhibitor. BJ

562 fibroblasts, WTC fibroblasts and U2OS cells were plated on standard $18 \mathrm{~mm}$ glass coverslips in

563 12-well cell culture plates prior to fixation.

564 For quantification of the pluripotency transcription factors OCT4 and NANOG, cells were

565 fixed with 3.5\% paraformaldehyde for 5 mins at room temperature, permeabilized with Tris-

566 buffered saline (TBS) with $0.1 \%$ Triton X-100 for $2 \times 5$ mins and blocked with TBS with $2 \%$

567 bovine serum albumin (BSA) and $0.1 \%$ Triton $\mathrm{X}-100$ for 30 mins at room temperature or

568 overnight at $4^{\circ} \mathrm{C}$. Primary antibodies were diluted in TBS $+2 \%$ BSA $+0.1 \%$ Triton $X-100$ at 5

$569 \mu \mathrm{g} / \mathrm{mL}$ (rabbit anti-OCT4, Abcam \#ab19857), 1:200 (mouse anti-NANOG, Abcam \#ab173368) or

570 1:150 (rabbit anti-NANOG, Abcam \#ab109250), and coverslips were incubated for 2 hrs at room

571 temperature. Cells were then washed with TBS $+2 \%$ BSA $+0.1 \%$ Triton $X-100$ for $4 \times 5$ mins.

572 Secondary antibodies were diluted in TBS $+2 \%$ BSA $+0.1 \%$ Triton X-100 $+0.5 \mu \mathrm{g} / \mathrm{mL}$ DAPI at

$573 \quad 1: 1000$ and coverslips were incubated for $1 \mathrm{~h}$ at room temperature. Cells were washed with TBS

$574+2 \%$ BSA $+0.1 \%$ Triton $\mathrm{X}-100$ for $2 \times 5$ mins, TBS $+0.1 \%$ Triton $\mathrm{X}-100$ for 5 mins and TBS

575 buffer for 5 mins sequentially. Coverslips were mounted on glass slides using ProLong Gold

576 antifade (ThermoFisher \#P36934) reagent. For SOX2 immunofluorescence, cells were fixed in

$5773.5 \%$ paraformaldehyde for 5 mins, washed $2 \times 5$ mins with TBS $+0.1 \%$ Triton X-100, blocked

578 with TBS $+2 \%$ BSA $+0.1 \%$ Triton X-100 $+10 \%$ donkey serum overnight at $4{ }^{\circ} \mathrm{C}$. Subsequently

579 the standard immunostaining protocol as described above was followed. The SOX2 primary

580 antibody was used at $10 \mu \mathrm{g} / \mathrm{mL}$ (mouse anti-SOX2, R\&D Systems \#MAB2018).

$581 \quad$ For measuring the frequency of chromosome segregation errors, cells were fixed with

582 ice-cold methanol for 5 mins and then permeabilized with high-salt TBS (containing $225 \mathrm{mM}$

$583 \mathrm{NaCl}$ ) with $0.1 \%$ Triton $\mathrm{X}-100$ for $2 \times 5$ mins and blocked with high-salt TBS with $2 \%$ BSA and 
$0.1 \%$ Triton $\mathrm{X}-100$ for 30 mins at room temperature or overnight at $4^{\circ} \mathrm{C}$. Primary antibodies were diluted in high-salt TBS $+2 \%$ BSA $+0.1 \%$ Triton $X-100$ at 1:4000 (mouse anti- $\alpha$-tubulin, Sigma \#T6199) and $2 \mu \mathrm{g} / \mathrm{mL}$ (rabbit anti-CENP-A, Dr. A. Straight. Stanford University). To assess calcium stable chromosome microtubule attachments, cells were pre-extracted with calcium buffer (100 mM PIPES, $1 \mathrm{mM} \mathrm{MgCl} 2,0.1 \mathrm{mM} \mathrm{CaCl}_{2}, 1 \%$ Triton $\mathrm{X}-100, \mathrm{pH}=6.8$ ) for 5 mins and subsequently fixed with $1 \%$ glutaraldehyde in PBS for 10 mins. Coverslips were washed with $0.5 \mathrm{mg} / \mathrm{mL}$ sodium borohydride ( $\mathrm{NaBH} 4$, dissolved in $\mathrm{PBS})$ for $2 \times 10$ mins and then rinsed with

591 PBS prior to blocking with TBS $+2 \%$ BSA $+0.5 \%$ Triton X-100 for 30 mins at room temperature.

592 Cells were stained with primary antibodies diluted with TBS $+2 \%$ BSA $+0.1 \%$ Triton X-100 at

593 1:1000 (human anti-ACA, Geisel School of Medicine at Dartmouth) and 1:4000 (mouse anti- $\alpha-$

594 tubulin, Sigma) following the standard immunostaining protocol as described above. The

595 following secondary antibodies (diluted at 1:1000) were used in this study: donkey anti-mouse 596 Alexa Fluor 488, goat anti-rabbit Alexa Fluor 594, donkey anti-mouse Alexa Fluor 647, donkey 597 goat anti-human Alexa Fluor 594, donkey anti-rabbit Alexa Fluor 647 (ThermoFisher \#A-21202, \#A-11037, \#A-31571, \#A-11014 and \#A-31573, respectively).

\section{Microscopy for Immunofluorescence}

Images were acquired with either a Hamamatsu ORCA-Fusion Gen III Scientific CMOS

602 camera mounted on a Nikon Eclipse Ti2E microscope with a Nikon CFI Plan Apo Lambda 60x,

6031.4 numerical aperture oil immersion objective, an Andor cooled CCD camera mounted on a

604 Nikon Ti microscope with a Nikon Plan Apo VC 60×, 1.4 numerical aperture oil immersion

605 objective or a spinning-disc confocal microscopy system (Micro Video Instruments) featuring a

606 Nikon Eclipse Ti microscope equipped with an Andor CSU-W1 two-camera spinning disc

607 module, Andor dual Zyla sCMOS cameras, an Andor ILE laser module, and a Nikon Plan Apo 
608 Lambda 60×, 1.4 numerical aperture oil immersion objective at room temperature. Image series

609 in the Z-axis were obtained using either $0.2 \mu \mathrm{m}$ or $0.5 \mu \mathrm{m}$ optical sections.

$610 \quad$ For experiments comparing the percentage of cells expressing a protein of interest or

611 quantifications of proteins levels, images for each cell line were acquired with the same

612 acquisition parameters and exposure times. Image deconvolution and contrast enhancement

613 were performed using NIS Batch Deconvolution (Nikon), NIS Elements (Nikon), ImageJ (NIH)

614 and Photoshop (Adobe). Images shown are maximum intensity projections (chromosome

615 segregation errors) or sum intensity projections (chromosome microtubule attachments and

616 pluripotency markers) of selected Z-planes.

617 Criteria for scoring chromosome segregation errors is as follows: the presence of a

618 chromosome that lags behind the segregating chromosomal mass and has clear centromere

619 staining in anaphase is scored as a lagging chromosome. The presence of a chromosome

620 without centromere staining between two segregating chromosomal masses in anaphase is

621 scored as an acentric DNA fragment. The presence of chromosome spanning between

622 segregating chromosomal masses in anaphase is scored as a chromosome bridge. A

623 chromosome that never aligns to the metaphase plane and presents proximal to the spindle

624 pole at anaphase onset is scored as an unaligned chromosome. An anaphase where

625 chromosomes segregate to more than two poles is scored as a multipolar anaphase. An

626 anaphase with multiple errors were scored as combination.

628 Quantification of Protein Expression

To determine the percentage of cells expressing a protein of interest, sum intensity projections were compiled from Z-stack images using ImageJ $(\mathrm{NIH})$. Then the maximum and

631 minimum display values were scaled equivalently among different cell lines using somatic cell

632 lines as a negative background control. Single cells were then categorized as positive or

633 negative for expression of a protein of interest. For quantification of protein levels, sum intensity 
634 projections were compiled from Z-stack images using ImageJ (NIH). Nuclei were randomly

635 picked per field of view based upon the DAPI signal. An elliptical region of interest (ROI) was

636 drawn to encompass the whole nucleus and then a slightly larger elliptical ROI was drawn to

637 encompass both the nucleus and the background. The mean background intensity was

638 calculated based on the in-between background region of the two ROls. Expression level of a

639 protein of interest in each nucleus was represented by the background subtracted mean

640 intensity of the ROI that encompasses the nucleus.

\section{Time-Lapse Live-Cell Fluorescence Imaging}

RPE-1 H2B-GFP cells were plated in standard DMEM media on $18 \mathrm{~mm}$ glass coverslips

in 12-well cell culture plates and incubated overnight at $37^{\circ} \mathrm{C}$ in a humidified atmosphere with

648 incubation, coverslips were washed into standard DMEM media supplemented with $100 \mu \mathrm{M}$

649 monastrol (Tocris \#1305) and maintained for $6 \mathrm{hrs}$ at $37^{\circ} \mathrm{C}$ in a humidified atmosphere with $5 \%$

$650 \mathrm{CO}_{2}$. After $6 \mathrm{hrs}$, cells were released by washing into phenol-free media supplemented with

$6510.1 \%$ DMSO, $3 \mu \mathrm{M}$ or $6 \mu \mathrm{M}$ proTAME (Tocris) and mounted in modified rose chambers. Live-

652 cell imaging was performed at $37^{\circ} \mathrm{C}$ using an Andor cooled CCD camera mounted on a Nikon $\mathrm{Ti}$

653 microscope with a Nikon Plan Apo VC 60×, 1.4 numerical aperture oil immersion objective with

654 binning set to $2 \times 2$. Image series in the Z-axis were obtained using $1 \mu \mathrm{m}$ optical sections. Cells

655 were imaged for $16 \mathrm{hrs}$ with a 2 min time interval for proTAME only experiments or 5 hrs with a

6562 min time interval for monastrol arrest and release experiments

657 H1 H2B-GFP hESCs and AICS-061 hiPSCs were plated in standard mTeSR1 media on

658 the $35 \mathrm{~mm}$ glass bottom dishes (MatTek \#P35G-1.5-14-C) coated with Matrigel or Laminin-521 
659 and incubated for $1-3$ days at $37^{\circ} \mathrm{C}$ in a humidified atmosphere with $5 \% \mathrm{CO}_{2}$ prior to live-cell

660 imaging. HPSCs were washed with phenol-free mTeSR1 media three times to get rid of spent

661 media and then cultured in phenol-free mTeSR1 during live-cell imaging. Live-cell imaging was

662 performed at $37^{\circ} \mathrm{C}$ in a humidified environment with $5 \% \mathrm{CO}_{2}$ (Tokai Hit Stage-top Incubation

663 System) using either a Hamamatsu ORCA-Fusion Gen III Scientific CMOS camera mounted on

664 a Nikon Eclipse Ti2E microscope with a Nikon CFI Plan Apo Lambda 60×, 1.4 numerical

665 aperture oil immersion objective with binning set to $2 \times 2$ or using spinning-disc confocal

666 microscopy system (Micro Video Instruments) featuring a Nikon Eclipse Ti microscope equipped

667 with an Andor CSU-W1 two-camera spinning disc module, Andor dual Zyla sCMOS cameras,

668 an Andor ILE laser module, and a Nikon Plan Apo Lambda 60×, 1.4 numerical aperture oil

669 immersion objective with binning set to $2 \times 2$. Image series in the Z-axis were obtained using 1

$670 \mu \mathrm{m}$ optical sections. HPSCs were imaged for 7 hours with 2 min time interval (wide-field

671 fluorescence) or 12 hours with 2 min time interval (spinning-disc confocal). Of note, we

672 optimized these experiments using the lowest exposure and intensity settings permissible to

673 visualize errors while minimizing artifacts due to phototoxicity.

Image acquisitions and analyses were performed using NIS Elements (Nikon) and

675 ImageJ $(\mathrm{NIH})$. Representative images from live-cell imaging shown in this study are maximum

676 intensity projections of all Z-planes or a single Z-plane. Cells undergoing mitosis were tracked

677 from nuclear envelope breakdown (NEB) to anaphase onset, during which prometaphase (NEB

678 to metaphase), metaphase (metaphase to anaphase onset) or total mitotic (NEB to anaphase

680 lagging chromosomes, chromosome bridges, multipolar anaphases, unaligned chromosomes,

681 or combinations of multiple errors were observed and scored. Criteria for scoring errors are

682 described in the immunofluorescence section. 
For immunofluorescence, cells were treated with 0.1\% DMSO, UMK57 or UMK95 (Dr. B

686 Kwok, University of Montreal) at the concentrations specified for 45 min prior to fixation. For

687 time-lapse live-cell imaging, hPSCs were cultured in phenol-free mTeSR1 supplemented with

$688 \quad 0.1 \%$ DMSO, $3 \mu \mathrm{M}, 6 \mu \mathrm{M}$ or $20 \mu \mathrm{M}$ proTAME (Tocris) or $2 \mu \mathrm{M}$ UMK57 or UMK95 during imaging. RPE-1 H2B-GFP cells were cultured in phenol-free media supplemented with $0.1 \%$

691 H2B-GFP cells were arrested in $100 \mu \mathrm{M}$ Monastol (Tocris) for $6 \mathrm{hrs}$ followed by washout with

692 phenol-free media into $0.1 \%$ DMSO, $3 \mu \mathrm{M}$ or $6 \mu \mathrm{M}$ proTAME (Tocris).

\section{All-trans Retinoic Acid Differentiation Assay}

H1 and H9 hESCs were plated on Matrigel-coated 18mm glass coverslips and grown in

698 including $1 \mu \mathrm{M}$ RA for 4 days prior to fixation. Daily morphological changes were monitored by bright-field phase contrast microscopy using a Hamamatsu ORCA $®$-Fusion Gen III Scientific

703 (Nikon) and ImageJ (NIH). Representative images shown in Supplementary Fig. 4 are from

704 selected single Z-plane that best illustrates the morphology.

\section{Statistics}


710 replicates. Significance was defined as ${ }^{*} p<0.05,{ }^{* *} p<0.01,{ }^{* * *} p<0.001,{ }^{* * * *} p<0.0001$. No

711 outliers were excluded in data analysis.

712

713 Acknowledgements:

714 We thank Jonathan Draper, Aaron Straight, Bruce Conklin, Benjamin Kwok, and Thorsten

715 Schlaeger for providing reagents or technical advice. We thank Ann Lavanway and the

716 Dartmouth College Life Sciences Light Microscopy Facility for assistance with microscopy. Also,

717 we thank members of the Compton and Godek laboratories for their helpful discussions and

718 comments, particularly Thomas Kucharski for his suggestions. This work was supported by

719 grant funding from the National Institutes of Health GM051542 to DAC and R01HD101436 to

720 KMG and a Hitchcock Foundation Pilot grant to KMG.

721

722

Author Contributions:

723 Conceptualization-KMG; Methodology- KMG, CD, and AY; Validation-KMG, CD and AY; Formal

724 Analysis-KMG, CD and AY; Investigation-KMG, CD and AY; Resources-KMG and DAC; Writing-

725 Original Draft- KMG and CD; Writing-Review and Editing- KMG, CD, DAC and AY;

726 Visualization-KMG and CD; Supervision-KMG; Funding Acquisition-KMG and DAC.

728 Declaration of Interests:

729 The authors declare no competing interests.

731 References:

733 Baart, E.B., Martini, E., Berg, I. van den, Macklon, N.S., Galjaard, R.-J.H., Fauser, B.C.J.M., and Opstal, 734 D.V. (2006). Preimplantation genetic screening reveals a high incidence of aneuploidy and mosaicism in 735 embryos from young women undergoing IVF. Human Reproduction 21, 223-233. 
Baker, D., Hirst, A.J., Gokhale, P.J., Juarez, M.A., Williams, S., Wheeler, M., Bean, K., Allison, T.F., Moore, H.D., Andrews, P.W., et al. (2016). Detecting Genetic Mosaicism in Cultures of Human Pluripotent Stem Cells. Stem Cell Reports 7, 998-1012.

Baker, D.E.C., Harrison, N.J., Maltby, E., Smith, K., Moore, H.D., Shaw, P.J., Heath, P.R., Holden, H., and Andrews, P.W. (2007). Adaptation to culture of human embryonic stem cells and oncogenesis in vivo. Nature Biotechnology 25, nbt1285.

Bakhoum, S.F., Thompson, S.L., Manning, A.L., and Compton, D.A. (2008). Genome stability is ensured by temporal control of kinetochore-microtubule dynamics. Nature Cell Biology 11, 27-35.

Bakhoum, S.F., Genovese, G., and Compton, D.A. (2009). Deviant Kinetochore Microtubule Dynamics Underlie Chromosomal Instability. Current Biology 19, 1937-1942.

Barroso-Vilares, M., Macedo, J.C., Reis, M., Warren, J.D., Compton, D., and Logarinho, E. (2020).

Becker, K.A., Ghule, P.N., Therrien, J.A., Lian, J.B., Stein, J.L., Wijnen, A.J. van, and Stein, G.S. (2006). Self-renewal of human embryonic stem cells is supported by a shortened G1 cell cycle phase. Journal of

751 Cellular Physiology 209, 883-893.

Bolton, H., Graham, S.J.L., Aa, N.V. der, Kumar, P., Theunis, K., Gallardo, E.F., Voet, T., and ZernickaGoetz, M. (2016). Mouse model of chromosome mosaicism reveals lineage-specific depletion of aneuploid cells and normal developmental potential. Nature Communications 7, 11165.

Burrell, R.A., McClelland, S.E., Endesfelder, D., Groth, P., Weller, M.-C., Shaikh, N., Domingo, E., cancer chromosomal instability. Nature 494, 492. J., Russell, J., Collins, T., et al. (2013). Lengthened G1 Phase Indicates Differentiation Status in Human

760 Embryonic Stem Cells. Stem Cells and Development 22, 279-295.

761 Cavazza, T., Takeda, Y., Politi, A.Z., Aushev, M., Aldag, P., Baker, C., Choudhary, M., Bucevičius, J., 762 Lukinavičius, G., Elder, K., et al. (2021). Parental genome unification is highly error-prone in mammalian 763 embryos. Cell 184, 2860-2877.e22.

764 Cimini, D., Tanzarella, C., and Degrassi, F. (1999). Differences in malsegregation rates obtained by 765 scoring ana-telophases or binucleate cells. Mutagenesis 14, 563-568.

766 Cimini, D., Howell, B., Maddox, P., and cell ..., K.-A. of (2001). Merotelic kinetochore orientation is a 767 major mechanism of aneuploidy in mitotic mammalian tissue cells.

768 Cimini, D., Moree, B., Canman, J.C., and Salmon, E.D. (2003). Merotelic kinetochore orientation occurs 769 frequently during early mitosis in mammalian tissue cells and error correction is achieved by two 770 different mechanisms. Journal of Cell Science 116, 4213-4225. 
Cimini, D., Wan, X., Hirel, C.B., and Salmon, E.D. (2006). Aurora Kinase Promotes Turnover of

773 Fragkos, M., and Naim, V. (2017). Rescue from replication stress during mitosis. Cell Cycle 16, 613-633.

774 Fragouli, E., Lenzi, M., Ross, R., Katz-Jaffe, M., Schoolcraft, W.B., and Wells, D. (2008).

775 Comprehensive molecular cytogenetic analysis of the human blastocyst stage. Human Reproduction 23,

$776 \quad 2596-2608$.

777

778

779

780

781

782

783

784

785

786

787

Fragouli, E., Alfarawati, S., Spath, K., Jaroudi, S., Sarasa, J., Enciso, M., and Wells, D. (2013). The origin and impact of embryonic aneuploidy. Human Genetics 132, 1001-1013.

Godek, K.M., and Compton, D.A. (2018). Quantitative methods to measure aneuploidy and chromosomal instability.

Godek, K.M., Kabeche, L., and Compton, D.A. (2014). Regulation of kinetochore-microtubule attachments through homeostatic control during mitosis. Nature Reviews Molecular Cell Biology 16, nrm3916.

Godek, K.M., Venere, M., Wu, Q., Mills, K.D., Hickey, W.F., Rich, J.N., and Compton, D.A. (2016). Chromosomal Instability Affects the Tumorigenicity of Glioblastoma Tumor-Initiating Cells. Cancer Discovery 6, 532-545.

Greenman, C., Stephens, P., Smith, R., Dalgliesh, G.L., Hunter, C., Bignell, G., Davies, H., Teague, J., Butler, A., Stevens, C., et al. (2007). Patterns of somatic mutation in human cancer genomes. Nature 446, 153.

Halliwell, J.A., Frith, T.J.R., Laing, O., Price, C.J., Bower, O.J., Stavish, D., Gokhale, P.J., Hewitt, Z., ElKhamisy, S.F., Barbaric, I., et al. (2020). Nucleosides Rescue Replication-Mediated Genome Instability of Human Pluripotent Stem Cells. Stem Cell Rep 14, 1009-1017.

Hassold, T., and Hunt, P. (2001). To err (meiotically) is human: the genesis of human aneuploidy. Nature Reviews Genetics 2, 35066065.

Hayashi, Y., Hsiao, E.C., Sami, S., Lancero, M., Schlieve, C.R., Nguyen, T., Yano, K., Nagahashi, A., Ikeya, M., Matsumoto, Y., et al. (2016). BMP-SMAD-ID promotes reprogramming to pluripotency by inhibiting p16/INK4A-dependent senescence. Proc National Acad Sci 113, 13057-13062.

Jain, A.K., Allton, K., Iacovino, M., Mahen, E., Milczarek, R.J., Zwaka, T.P., Kyba, M., and Barton, M.C. (2012). p53 Regulates Cell Cycle and MicroRNAs to Promote Differentiation of Human Embryonic Stem Cells. PLoS Biology 10, e1001268.

Keller, A., and Spits, C. (2021). The Impact of Acquired Genetic Abnormalities on the Clinical Translation of Human Pluripotent Stem Cells. Cells 10, 3246.

Knouse, K.A., Wu, J., Whittaker, C.A., and Amon, A. (2014). Single cell sequencing reveals low levels of aneuploidy across mammalian tissues. Proceedings of the National Academy of Sciences 111, 1340913414. 
Knouse, K.A., Lopez, K.E., Bachofner, M., and Amon, A. (2018). Chromosome Segregation Fidelity in Epithelia Requires Tissue Architecture. Cell.

Lamm, N., Ben-David, U., Golan-Lev, T., Storchová, Z., Benvenisty, N., and Kerem, B. (2016). Genomic Instability in Human Pluripotent Stem Cells Arises from Replicative Stress and Chromosome Condensation Defects. Cell Stem Cell 18, 253-261.

Lightfoot, D.A., Kouznetsova, A., Mahdy, E., Wilbertz, J., and Höög, C. (2006). The fate of mosaic aneuploid embryos during mouse development. Developmental Biology 289, 384-394.

Macklon, N., Geraedts, J., and Fauser, B. (2002). Conception to ongoing pregnancy: the "black box" of early pregnancy loss. Human Reproduction Update 8, 333-343.

Mayshar, Y., Ben-David, U., Lavon, N., Biancotti, J.-C., Yakir, B., Clark, A.T., Plath, K., Lowry, W.E., and Benvenisty, N. (2010). Identification and Classification of Chromosomal Aberrations in Human Induced Pluripotent Stem Cells. Cell Stem Cell 7, 521-531.

McCoy, R.C. (2017). Mosaicism in Preimplantation Human Embryos: When Chromosomal Abnormalities Are the Norm. Trends in Genetics 33, 448-463.

McCoy, R.C., Demko, Z.P., Ryan, A., Banjevic, M., Hill, M., Sigurjonsson, S., Rabinowitz, M., and Petrov, D.A. (2015). Evidence of Selection against Complex Mitotic-Origin Aneuploidy during Preimplantation Development. PLOS Genetics 11, e1005601.

Menasha, J., Levy, B., Hirschhorn, K., and Kardon, N.B. (2005). Incidence and spectrum of chromosome abnormalities in spontaneous abortions\&colon; New insights from a 12-year study. Genetics in Medicine 7, 251-263.

Merkle, F.T., Ghosh, S., Kamitaki, N., Mitchell, J., Avior, Y., Mello, C., Kashin, S., Mekhoubad, S., Ilic, D., Charlton, M., et al. (2017). Human pluripotent stem cells recurrently acquire and expand dominant negative P53 mutations. Nature 545, 229.

Mertzanidou, A., Wilton, L., Cheng, J., Spits, C., Vanneste, E., Moreau, Y., Vermeesch, J.R., and Sermon, K. (2013). Microarray analysis reveals abnormal chromosomal complements in over 70\% of 14 normally developing human embryos. Human Reproduction 28, 256-264.

Milagre, I., Pereira, C., Oliveira, R.A., and Jansen, L.E.T. (2020). Reprogramming of human cells to pluripotency induces CENP-A chromatin depletion. Open Biol 10, 200227.

Musacchio, A., and Salmon, E.D. (2007). The spindle-assembly checkpoint in space and time. Nature Reviews Molecular Cell Biology 8, 379-393.

Nath, S., Ghatak, D., Das, P., and Roychoudhury, S. (2015). Transcriptional Control of Mitosis: Deregulation and Cancer. Front Endocrinol 6, 60.

Orr, B., Godek, K.M., and Compton, D. (2015). Aneuploidy. Current Biology 25, R538-R542.

Orr, B., Talje, L., Liu, Z., Kwok, B.H., and Compton, D.A. (2016). Adaptive Resistance to an Inhibitor of Chromosomal Instability in Human Cancer Cells. Cell Reports 17, 1755-1763. 
Passerini, V., Ozeri-Galai, E., Pagter, M.S. de, Donnelly, N., Schmalbrock, S., Kloosterman, W.P., Kerem, B., and Storchová, Z. (2016). The presence of extra chromosomes leads to genomic instability. Nature Communications 7, 10754.

Peterson, S.E., Westra, J.W., Rehen, S.K., Young, H., Bushman, D.M., Paczkowski, C.M., Yung, Y.C., Lynch, C.L., Tran, H.T., Nickey, K.S., et al. (2011). Normal Human Pluripotent Stem Cell Lines Exhibit Pervasive Mosaic Aneuploidy. PLoS ONE 6, e23018.

Popovic, M., Dhaenens, L., Taelman, J., Dheedene, A., Bialecka, M., Sutter, P.D., Lopes, S.M.C. de S., Menten, B., and Heindryckx, B. (2019). Extended in vitro culture of human embryos demonstrates the complex nature of diagnosing chromosomal mosaicism from a single trophectoderm biopsy. Human Reproduction (Oxford, England).

Price, C.J., Stavish, D., Gokhale, P.J., Stevenson, B.A., Sargeant, S., Lacey, J., Rodriguez, T.A., and Barbaric, I. (2021). Genetically variant human pluripotent stem cells selectively eliminate wild-type counterparts through YAP-mediated cell competition. Dev Cell 56, 2455-2470.e10.

Salimian, K.J., Ballister, E.R., Smoak, E.M., Wood, S., Panchenko, T., Lampson, M.A., and Black, B.E. (2011). Feedback Control in Sensing Chromosome Biorientation by the Aurora B Kinase. Current Biology 21, 1158-1165.

Sansregret, L., Patterson, J.O., Dewhurst, S., López-García, C., Koch, A., McGranahan, N., Chao, W.C.H., Barry, D.J., Rowan, A., Instrell, R., et al. (2017). APC/C Dysfunction Limits Excessive Cancer Chromosomal Instability. Cancer Discovery 7, 218-233.

Sheltzer, J.M., Blank, H.M., Pfau, S.J., Tange, Y., George, B.M., Humpton, T.J., Brito, I.L., Hiraoka, Y., Niwa, O., and Amon, A. (2011). Aneuploidy Drives Genomic Instability in Yeast. Science 333, 10261030 .

Stingele, S., Stoehr, G., Peplowska, K., Cox, J., Mann, M., and Storchova, Z. (2012). Global analysis of genome, transcriptome and proteome reveals the response to aneuploidy in human cells. Molecular Systems Biology 8, 608 .

Taapken, S.M., Nisler, B.S., Newton, M.A., Sampsell-Barron, T.L., Leonhard, K.A., McIntire, E.M., and Montgomery, K.D. (2011). Karyotypic abnormalities in human induced pluripotent stem cells and embryonic stem cells. Nature Biotechnology 29, 313.

Thompson, S.L., and Compton, D.A. (2008). Examining the link between chromosomal instability and aneuploidy in human cells. The Journal of Cell Biology 180, 665-672.

Thompson, S.L., and Compton, D.A. (2010). Proliferation of aneuploid human cells is limited by a p53dependent mechanism. The Journal of Cell Biology 188, 369-381.

Thompson, S.L., Bakhoum, S.F., and Compton, D.A. (2010). Mechanisms of Chromosomal Instability. Current Biology 20, R285-R295.

Thomson, J.A., Itskovitz-Eldor, J., Shapiro, S.S., Waknitz, M.A., Swiergiel, J.J., Marshall, V.S., and Jones, J.M. (1998). Embryonic Stem Cell Lines Derived from Human Blastocysts. Science 282, 11451147. 
Vanneste, E., Voet, T., Caignec, C., Ampe, M., Konings, P., Melotte, C., Debrock, S., Amyere, M., Vikkula, M., Schuit, F., et al. (2009). Chromosome instability is common in human cleavage-stage embryos. Nature Medicine 15, nm.1924.

Vázquez-Diez, C., Paim, L.M.G.M., and FitzHarris, G. (2019). Cell-Size-Independent Spindle Checkpoint Failure Underlies Chromosome Segregation Error in Mouse Embryos. Current Biology : CB.

Warren, J.D., Orr, B., and Compton, D.A. (2020). A comparative analysis of methods to measure kinetochore-microtubule attachment stability. Methods Cell Biol 158, 91-116.

Wei, Y., Multi, S., Yang, C.-R., Ma, J., Zhang, Q.-H., Wang, Z.-B., Li, M., Wei, L., Ge, Z.-J., Zhang, C.H., et al. (2011). Spindle Assembly Checkpoint Regulates Mitotic Cell Cycle Progression during Preimplantation Embryo Development. PLoS ONE 6, e21557.

Yang, M., Rito, T., Metzger, J., Naftaly, J., Soman, R., Hu, J., Albertini, D.F., Barad, D.H., Brivanlou, A.H., and Gleicher, N. (2021). Depletion of aneuploid cells in human embryos and gastruloids. Nat Cell Biol 23, 314-321.

Zeng, X., Sigoillot, F., Gaur, S., Choi, S., Pfaff, K.L., Oh, D.-C.C., Hathaway, N., Dimova, N., Cuny, G.D., and King, R.W. (2010). Pharmacologic inhibition of the anaphase-promoting complex induces a spindle checkpoint-dependent mitotic arrest in the absence of spindle damage. Cancer Cell 18, 382-395.

Zhang, J., Hirst, A.J., Duan, F., Qiu, H., Huang, R., Ji, Y., Bai, L., Zhang, F., Robinson, D., Jones, M., et al. (2019). Anti-apoptotic Mutations Desensitize Human Pluripotent Stem Cells to Mitotic Stress and Enable Aneuploid Cell Survival. Stem Cell Reports.

Zhao, Y., Carter, R., Natarajan, S., Varn, F.S., Compton, D.A., Gawad, C., Cheng, C., and Godek, K.M. (2019). Single-cell RNA sequencing reveals the impact of chromosomal instability on glioblastoma cancer stem cells. BMC Medical Genomics 12, 79.

\section{Figure Legends:}

Figure 1. Mitotic error rates are elevated in hPSCs compared to somatic cells. (A)

Representative images of anaphase errors including a lagging chromosome with a centromere

(white arrow), chromosome bridge, acentric DNA fragment lacking a centromere (box), unaligned chromosome with a centromere (yellow arrowhead) and multipolar anaphase in $\mathrm{H} 1$ hESCs. Shown is DNA (cyan), centromeres (magenta) and microtubules (yellow). Scale bars: 5 $\mu \mathrm{m}$. (B) Percentage of anaphase errors in primary somatic BJ fibroblasts and $\mathrm{H} 1$ and $\mathrm{H} 9 \mathrm{hESCs}$ plated as single cells on Laminin-521. $n=317$ (BJ fibroblasts), 251 (H1 hESCs), and 283 (H9 hESCs) anaphases from three independent experiments; ${ }^{*} p<0.05,{ }^{* *} p<0.01,{ }^{* * *} p<0.0001$ 
910 using a two-tailed Fisher's exact test. (C) Representative images of chromosome microtubule

911 attachments in metaphase and anaphase H1 hESCs. Shown is DNA (cyan), microtubules

912 (yellow) and centromeres (magenta). In the metaphase cell, boxes are pairs of centromeres with

913 a bioriented attachment (dashed) or a merotelic attachment (solid, white arrow). The anaphase

914 cell shows a lagging chromosome with a merotelic attachment (white arrow). Insets are

915 magnified views. Scale bars: $5 \mu \mathrm{m}$ (main) and $1 \mu \mathrm{m}$ (insets). (D) Selected panels from time-

916 lapse live-cell fluorescence imaging of H1 H2B-GFP hESCs showing a normal anaphase and

917 erroneous anaphases with a lagging chromosome (white arrow) or chromosome bridge (yellow

918 arrow). Scale bar: $10 \mu \mathrm{m}$. (E) Percentage of anaphase errors in somatic RPE-1 H2B-GFP, H1

919 H2B-GFP hESCs plated as single cells on Laminin-521 or as aggregates on Matrigel and AICS-

920061 hiPSCs. $n=46$ anaphases in RPE-1 and $n=111(H 1$ on Laminin-521), 121 (H1 on

921 Matrigel), and 258 (AICS-061) anaphases in hPSCs from at least three independent

922 experiments; ${ }^{*} p<0.05$ using a two-tailed Fisher's exact test. See also Figure S1.

924 Figure 2. Prolonging mitotic duration decreases mitotic error rates in hPSCs. (A) Model

925 illustrating the relationship between mitotic errors and mitotic duration. Early in mitosis improper

926 chromosome microtubule attachments are prevalent due to the stochastic interaction of

927 microtubules with chromosomes, but errors decline as mitosis progresses and improper

928 attachments are converted to correct ones. Prolonging mitosis using the small molecule

929 proTAME increases the amount of time for microtubule error correction reducing the frequency

930 of mitotic errors. (B) Selected panels from time-lapse live-cell fluorescence imaging of $\mathrm{H} 1 \mathrm{H} 2 \mathrm{~B}-$

931 GFP hESCs that were treated with DMSO or increasing concentrations of proTAME (yellow

932 arrowheads indicate daughter nuclei). Scale bar: $10 \mu \mathrm{m}$. (C, D) Mitotic duration (C) and

933 percentage of lagging chromosomes or chromosome bridges (D) in H1 H2B-GFP hESCs

934 treated with DMSO or increasing concentrations of proTAME. $\mathrm{n}=275$ (DMSO), 247 (3 $\mu \mathrm{M}$ 
935 proTAME), 250 ( $6 \mu \mathrm{M}$ proTAME), and 257 (20 $\mu \mathrm{M}$ proTAME) anaphases from six independent

936 experiments; NEB: nuclear envelope breakdown; AO: anaphase onset; mean \pm SD and ${ }^{* * * *} p<$

9370.0001 using a one-way ANOVA and Dunnett's multiple comparisons test (C); n.s. $p>0.05,{ }^{*} p<$

$9380.05,{ }^{* *} p<0.01,{ }^{* * *} p<0.001$ using a two-tailed Fisher's exact test $(\mathbf{C})$. See also Figure S2.

Figure 3. Decreasing microtubule attachment stability reduces mitotic errors in hPSCs.

941 (A) Model illustrating the relationship between mitotic errors and chromosome microtubule

942 attachment stability. Higher microtubule attachment stability decreases the correction rate of

943 improper attachments while lowering microtubule stability using the small molecule UMK57

944 increases the correction rate of improper attachments reducing mitotic errors. (B) Percentage of

945 lagging chromosomes and chromosome bridges in $\mathrm{H} 1$ and $\mathrm{H} 9 \mathrm{hESC}$ and U2OS cancer cells

946 after treatment with DMSO, UMK57 or UMK95 an inactive analog of UMK57 for 45 mins. $\mathrm{n}=$

947431 (DMSO), 390 (1 4 M UMK57), 391 (2 $4 \mathrm{M}$ UMK57), and 410 (2 $\mu \mathrm{M}$ UMK95) anaphases in H1

948 hESCs. $\mathrm{n}=405$ (DMSO), 385 (1 $\mu \mathrm{M}$ UMK57), 367 (2 $\mu \mathrm{M}$ UMK57), and 393 (2 $\mu \mathrm{M}$ UMK95)

949 anaphases in H9 hESCs. $\mathrm{n}=199$ (DMSO), 242 (0.1 $\mu$ M UMK57), and 243 (0.1 $\mu$ M UMK95)

950 anaphases in U2OS from three independent experiments; n.s. $p>0.05,{ }^{*} p<0.05,{ }^{* *} p<0.01$,

$951^{* * * *} p<0.0001$ using a two-tailed Fisher's exact test. (C, D) Percentage of lagging chromosomes

952 and chromosome bridges from time-lapse live-cell fluorescence imaging of H1 H2B-GFP hESCs

953 (C) or AICS-061 hiPSCs (D) treated with DMSO, UMK57 or UMK95 for 12 hrs. n = $205(\mathrm{H} 1$,

954 DMSO), 278 (H1, $2 \mu \mathrm{M}$ UMK57), 187 (H1, $2 \mu \mathrm{M}$ UMK95), 158 (AICS-061, DMSO), 209 (AICS-

955 061, $2 \mu \mathrm{M}$ UMK57), and 177 (AICS-061, $2 \mu \mathrm{M}$ UMK95) anaphases from three independent

956 experiments; n.s. $p>0.05,{ }^{*} p<0.05,{ }^{* *} p<0.01$ using a two-tailed Fisher's exact test. See also

957 Figure S3.

958 
959 Figure 4. Developmental potential influences mitotic error rates. (A) Model illustrating the

960 correlation between mitotic errors and developmental potential. As developmental potential

961 decreases mitotic errors decrease, and conversely as developmental potential increases mitotic

962 errors increase. (B) Percentage of lagging chromosomes and chromosome bridges in isogenic

963 somatic WTC fibroblasts, WTC-11 hiPSCs and AICS-061 hiPSCs. $n=268$ (WTC fibroblasts),

964421 (WTC-11 hiPSCs), and 438 (AICS-061 hiPSCs) anaphases from three independent

965 experiments; ${ }^{* *} p<0.001,{ }^{* * * *} p<0.0001$ using a two-tailed Fisher's exact test. (C) Percentage

966 of lagging chromosomes and chromosome bridges in $\mathrm{H} 1$ and $\mathrm{H} 9 \mathrm{hESC}$ after 4 day treatment

967 with DMSO or $1 \mu \mathrm{M}$ all-trans retinoic acid $(\mathrm{RA})$ to induce undirected differentiation. $\mathrm{n}=454(\mathrm{H} 1$,

968 DMSO), 358 (H1, $1 \mu \mathrm{M} \mathrm{RA}), 398$ (H9, DMSO), and 356 (H9, $1 \mu \mathrm{M} \mathrm{RA})$ anaphases from three

969 independent experiments; ${ }^{*} p<0.05,{ }^{* * *} p<0.001,{ }^{* * *} p<0.0001$ using a two-tailed Fisher's

970 exact test. See also Figures S4 and S5.

972 Figure 5. Models for how mitotic errors contribute to culture adaptation in hPSCs and

973 aneuploidy during human development. (A) We speculate that culture adaptation in hPSCs

974 depends on lagging chromosome errors that lead to chromosome missegregations and the

975 generation of aneuploid progeny. Most aneuploid hPSCs (orange) are at a growth disadvantage

976 and are outcompeted by diploid hPSCs (blue) as chromosome missegregation is random with

977 respect to which chromosome is missegregated. However, the persistent and high rate of

978 lagging chromosome errors in hPSCs coupled with continued propagation in culture increases

979 the probability that aneuploid hPSCs with stable chromosome abnormalities conferring a growth

980 advantage over diploid hPSCs are selected for (purple hPSCs). (B) We propose that

981 preimplantation totipotent and pluripotent embryonic cells exhibit a high rate of lagging

982 chromosomes that cause chromosome segregation errors and the generation of aneuploid

983 embryonic cells leading to mosaic embryos composed of mixed populations of diploid and 
bioRxiv preprint doi: https://doi.org/10.1101/2022 03.01482524 ; this version posted March 1, 2022. The copyright holder for this preprint (which was not certified by peer review) is the author/funder, who has granted bioRxiv a license to display the preprint in perpetuity. It is made available under aCC-BY-NC-ND 4.0 International license.

984 aneuploid embryonic cells. However, as development progresses, developmental potential

985 decreases coinciding with a decline in the lagging chromosome rate that when coupled with a

986 selective disadvantage for aneuploid (orange) compared to diploid (blue) embryonic cells

987 explains how mosaic embryos can support normal human development. 


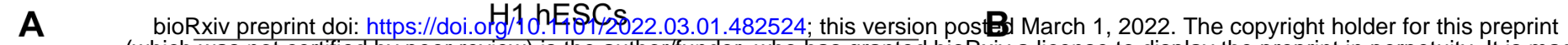

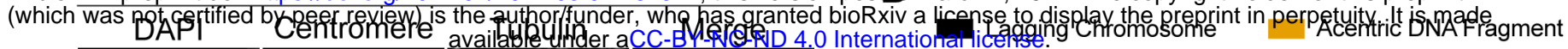

Lagging

Chromosome

Chromosome Bridge

Acentric DNA

Fragment

\section{Unaligned} Chromosome

Multipolar

Anaphase

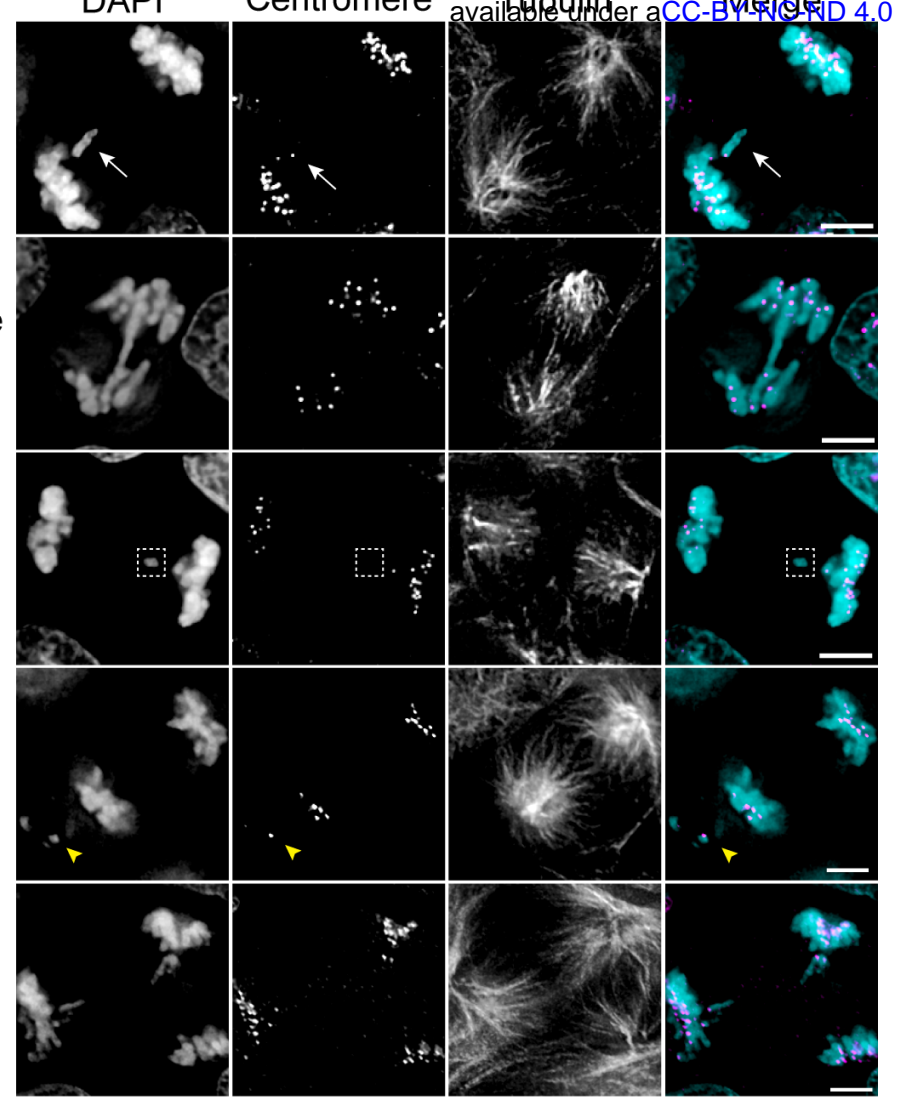

Chromosome Bridge Multipolar Anaphase

Unaligned Chromosome Combination

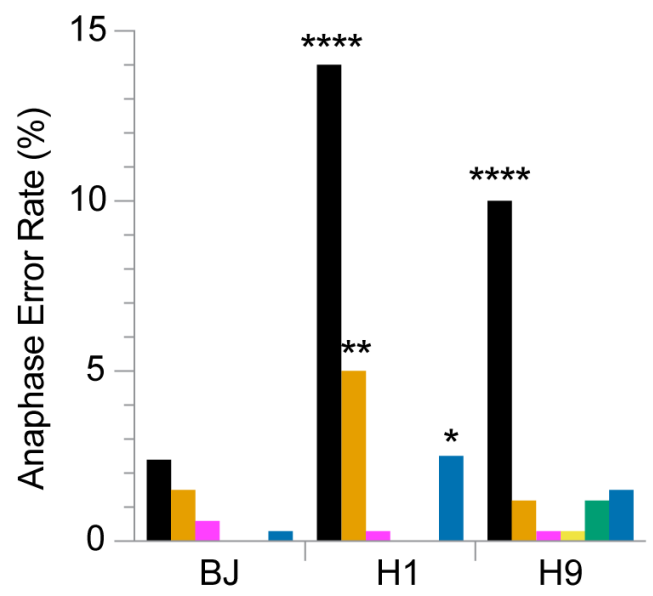

Fibroblasts hESCs hESCs

C

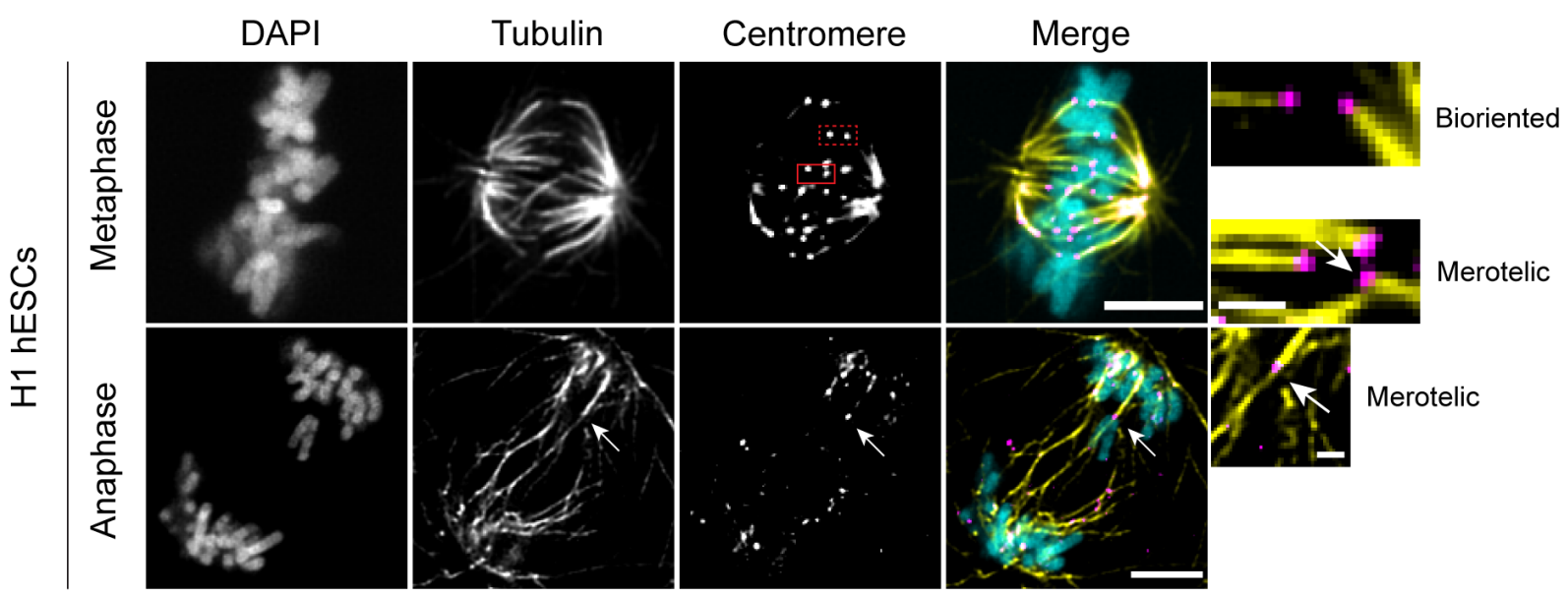

D

H1 H2B-GFP hESCs

\begin{tabular}{ccc}
\hline NEB & Metaphase & Anaphase \\
$0 \mathrm{~min}$ & $12 \mathrm{~min}$ & $28 \mathrm{~min}$ \\
$\mathrm{~m}$ & $\mathbf{3 0} \mathrm{min}$
\end{tabular}

Normal

Anaphase

Lagging

Chromosome

Chromosome Bridge
E
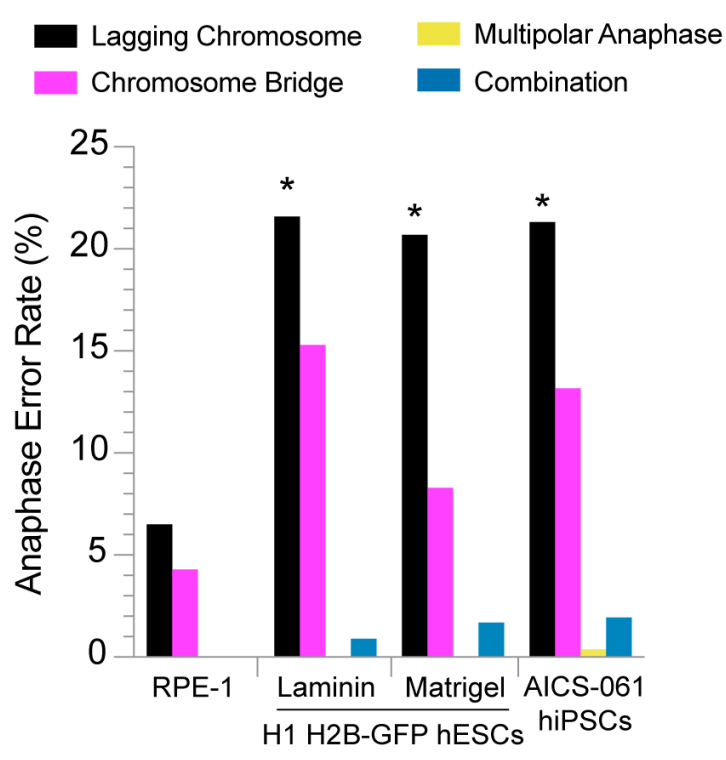
A bioRxiv preprint doi: https://doi.org/10.1101/2022.03.01 (which was not certified by peer review) is the author/funder, who has granted bioRxiv a license to display the preprint in perpetuity. It is made

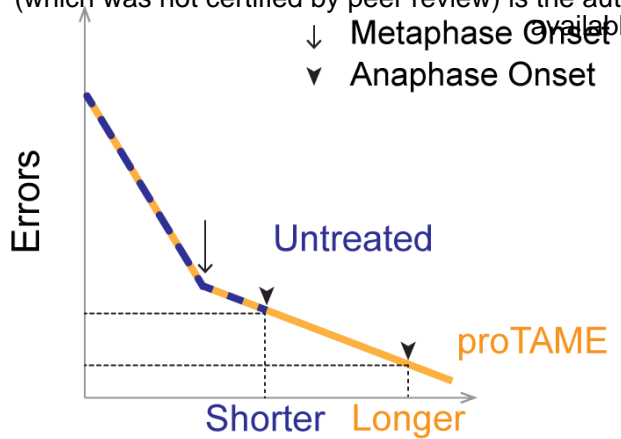

Mitotic Duration

$\begin{array}{ll}\text { C } & \text { Normal Anaphase } \quad \text { Lagging Chromosome } \\ & \text { Chromosome Bridge Multipolar Anaphase } \\ \text { Unaligned Chromosome } & \end{array}$

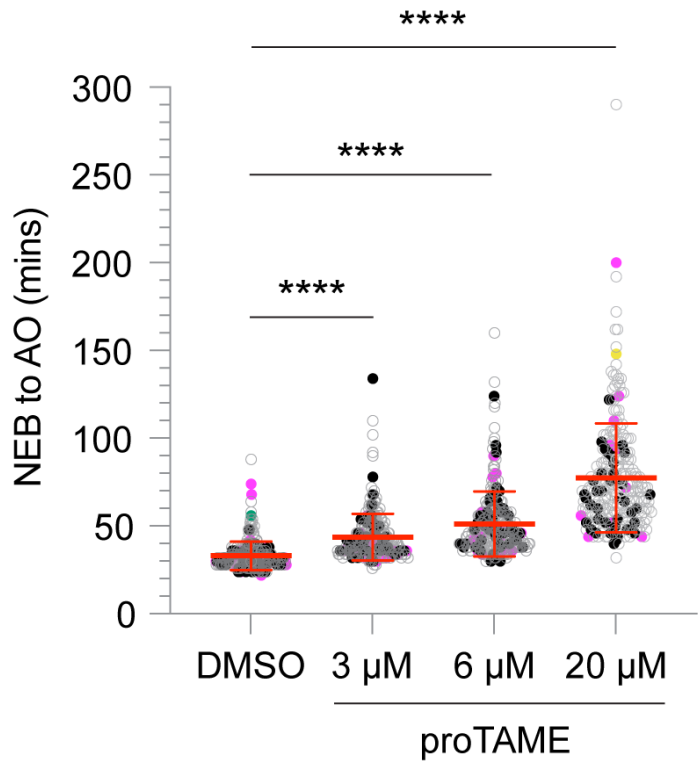

D Lagging Chromosome $\square$ Chromosome Bridge

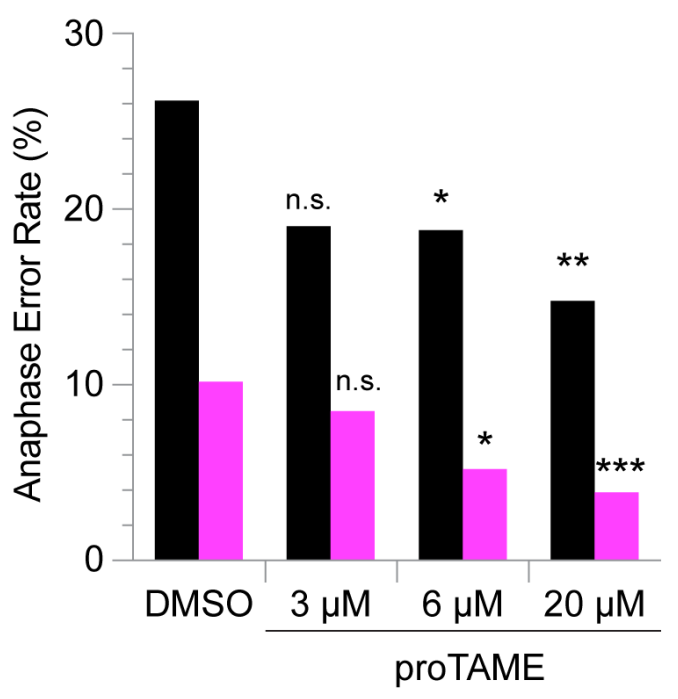

DMSO proTAME proTAME proTAME

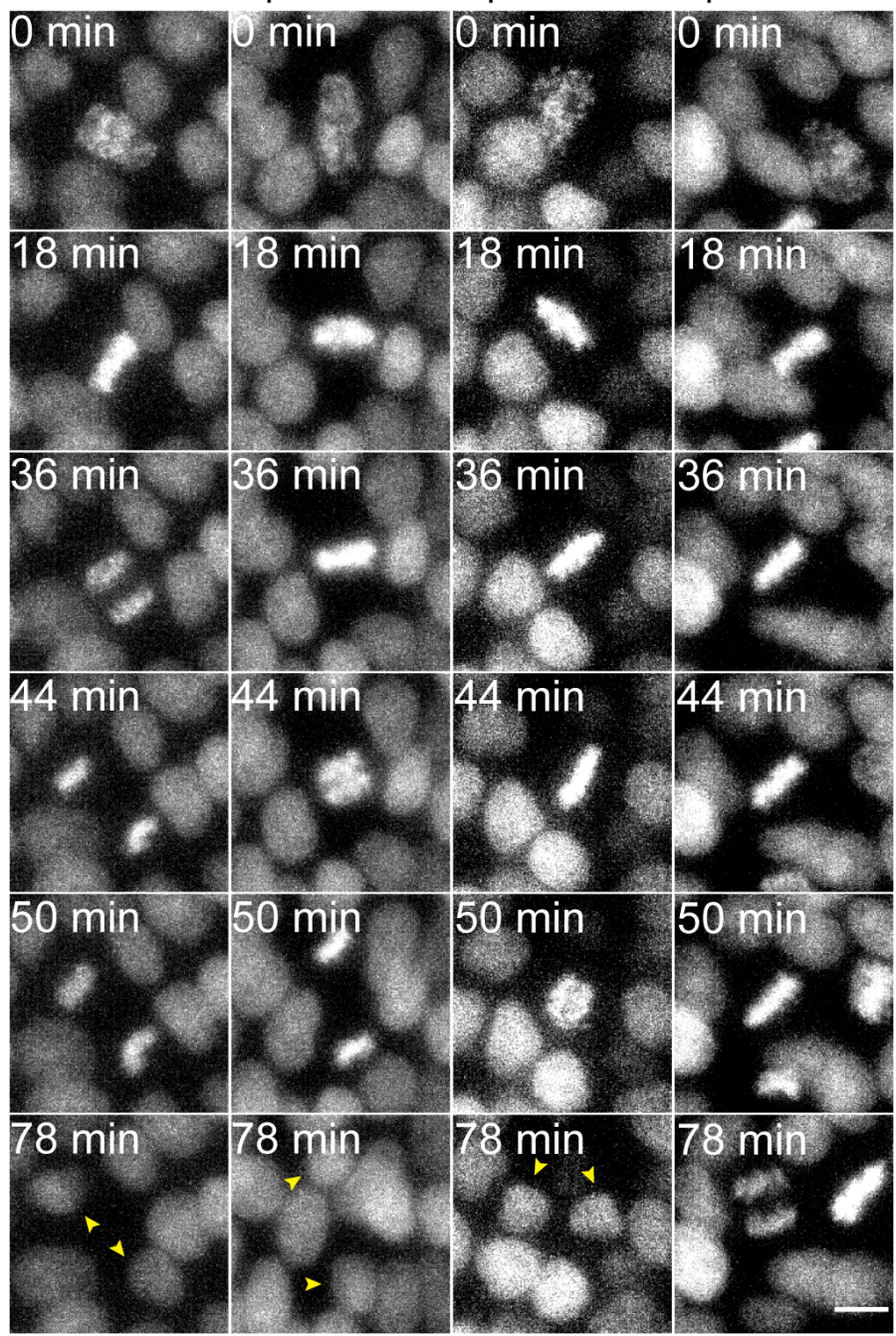



(which was not certified by peer review) is the author/funder, who has granted bionxivatieense to display the preprint in perpetuity. It is made available under aCC-BY-NC-ND 4.0 International license.

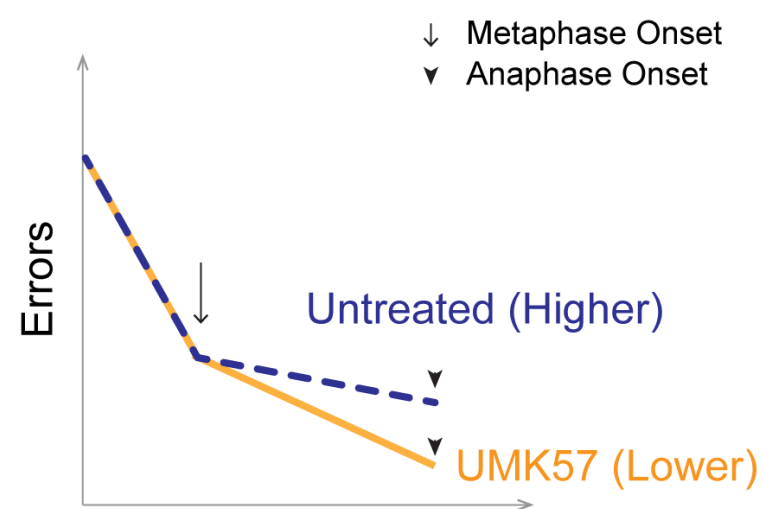

Mitotic Duration
C

- Lagging Chromosome $\square$ Chromosome Bridge

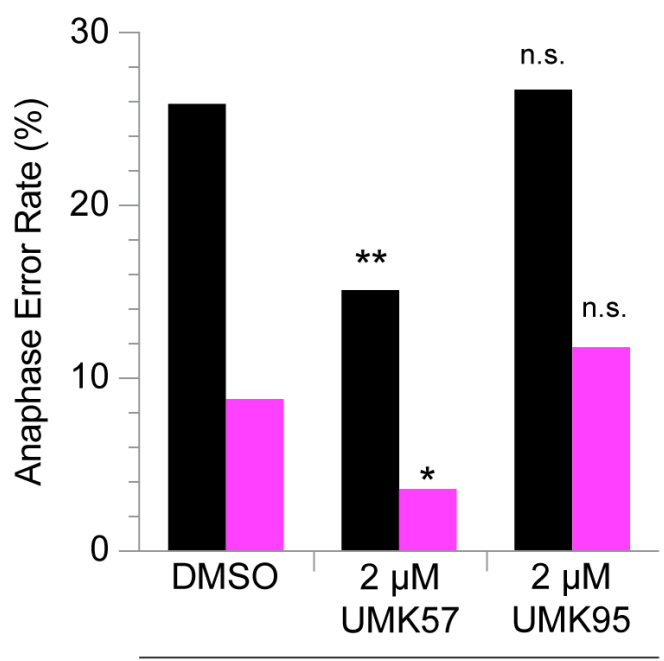

H1 H2B-GFP hESCs

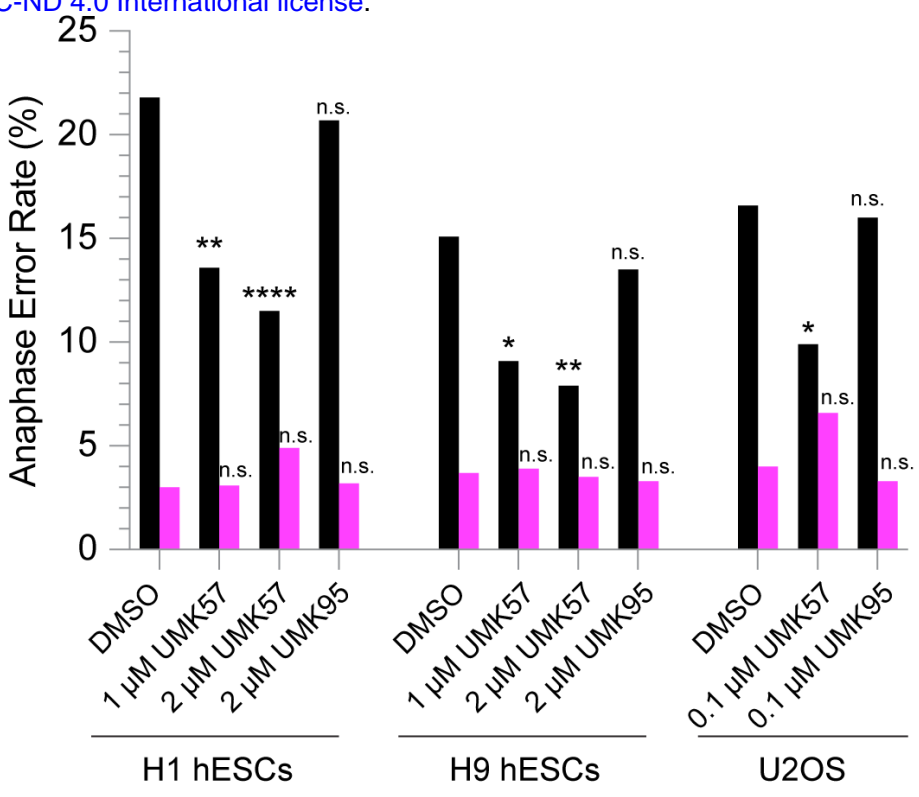

- Lagging Chromosome $\square$ Chromosome Bridge

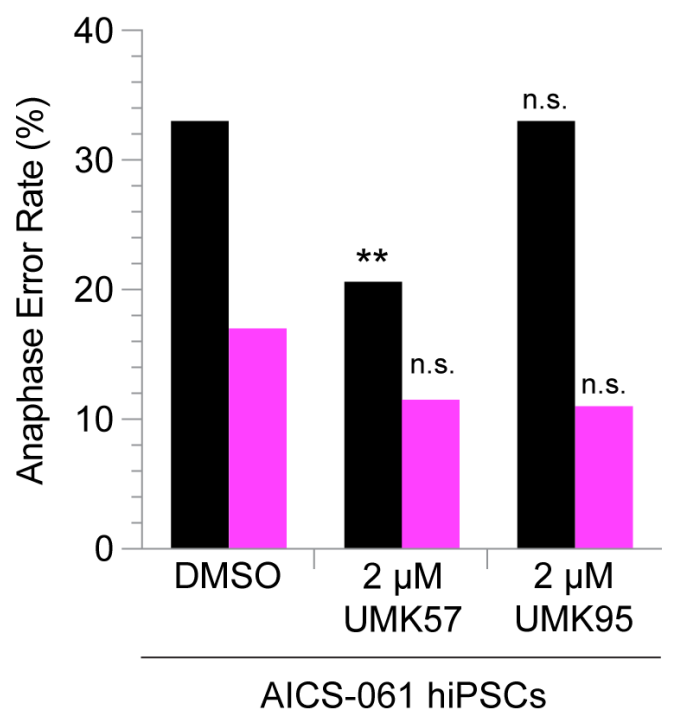


A

\section{Human Pluripotent Stem Cells}

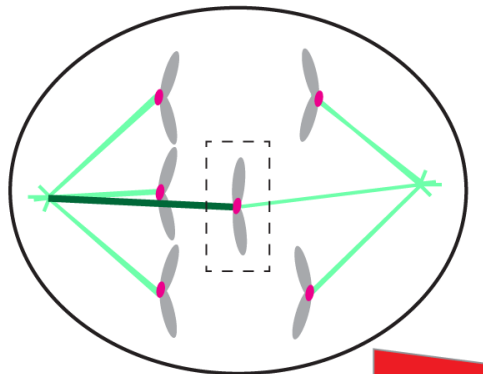

Merotelic attachment Bioriented attachment

\section{HIGH}

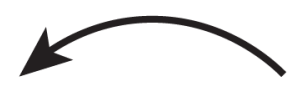

Reprogramming

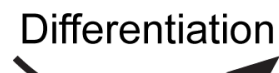

Human Somatic Cells

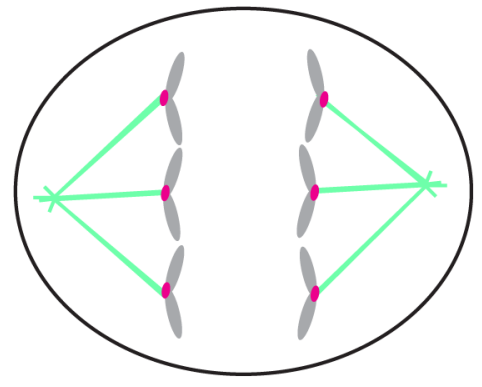

Mitotic Error Rates

\section{LOW}

B

Lagging Chromosome Chromosome Bridge

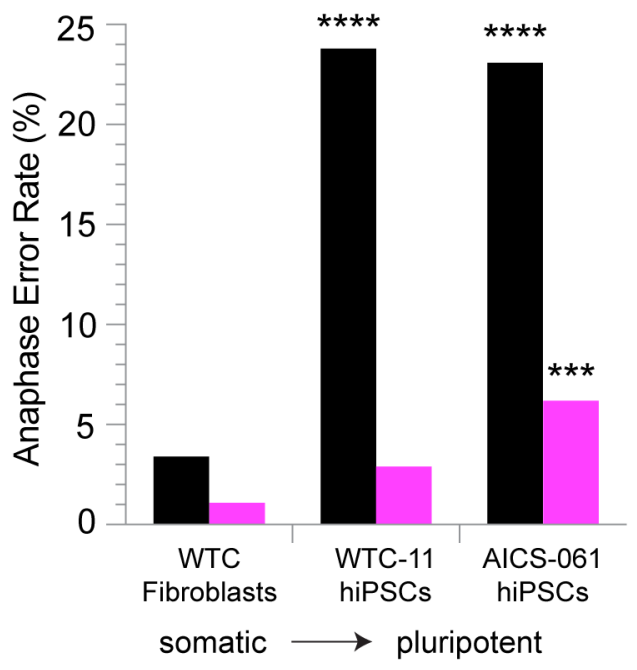

C

- Lagging Chromosome Chromosome Bridge

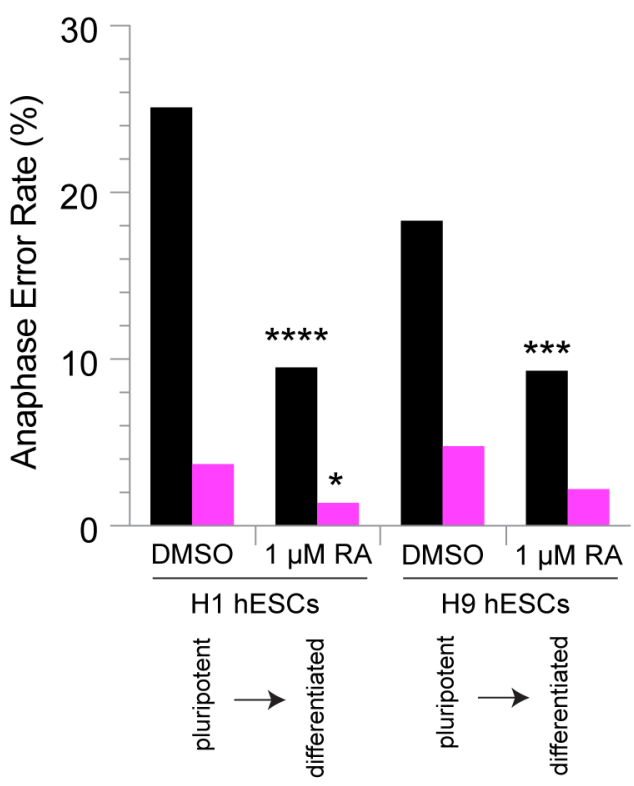


